\title{
Verifiable Inner Product Encryption Scheme
}

\author{
Najmeh Soroush ${ }^{1(\bowtie)}$, Vincenzo Iovino ${ }^{1,2}$, Alfredo Rial ${ }^{1}$, Peter B. Roenne ${ }^{1}$, \\ and Peter Y. A. Ryan ${ }^{1}$ \\ 1 SnT, University of Luxembourg, Luxembourg City, Luxembourg \\ \{najmeh.soroush, alfredo.rial, peter.roenne, peter.ryan\}@uni.lu \\ 2 University of Salerno, Salerno, Italy \\ vinciovino@gmail.com
}

\begin{abstract}
In the standard setting of functional encryption (FE), we assume both the Central Authority (CA) and the encryptors to run their respective algorithms faithfully. Badrinarayanan et al. [ASIACRYPT 2016] proposed the concept of verifiable FE, which essentially guarantees that dishonest encryptors and authorities, even when colluding together, are not able to generate ciphertexts and tokens that give "inconsistent" results. They also provide a compiler turning any perfectly correct FE into a verifiable $\mathrm{FE}$, but do not give efficient constructions.

In this paper we improve on this situation by considering InnerProduct Encryption (IPE), which is a special case of functional encryption and a primitive that has attracted wide interest from both practitioners and researchers in the last decade. Specifically, we construct the first efficient verifiable IPE (VIPE) scheme according to the innerproduct functionality of Katz, Sahai and Waters [EUROCRYPT 2008]. To instantiate the general construction of Badrinarayanan et al. we need to solve several additional challenges. In particular, we construct the first efficient perfectly correct IPE scheme. Our VIPE satisfies unconditional verifiability, whereas its privacy relies on the DLin assumption.
\end{abstract}

Keywords: Inner-product encryption · Verifiability $\cdot$ Functional commitments

\section{Introduction}

Functional encryption (FE) is a new encryption paradigm that was first proposed by Sahai and Waters [23] and formalized by Boneh, Sahai and Waters [7]. Informally, in an FE system, a decryption key allows a user to learn a function of the original message. More specifically, in a FE scheme for functionality $F: K \times \mathcal{M} \rightarrow \mathcal{C} \mathcal{T}$, defined over key space $\mathcal{K}$, message space $\mathcal{M}$ and output space $\mathcal{C} \mathcal{T}$, for every key $k \in K$, the owner of the master secret key MSK associated with master public key MPK can generate a token $\mathrm{Tok}_{k}$ that allows the computation of $F(k, m)$ from a ciphertext of $x$ computed under the master public key MPK.

This research were supported by the Luxembourg National Research Fund (FNR).

(C) International Association for Cryptologic Research 2020

A. Kiayias et al. (Eds.): PKC 2020, LNCS 12110, pp. 65-94, 2020.

https://doi.org/10.1007/978-3-030-45374-9_3 
A notable special case of FE is that of inner product encryption (IPE). In IPE $[8,18,19,21,22]$ the message is a pair $(m, \boldsymbol{x})$, with $m \in \mathcal{M}$, the payload message and $\boldsymbol{x}$ is an attribute vector in the set $\Sigma$ and the token is associated with a vector $\boldsymbol{v} \in \Sigma$. The functionality is $F(\boldsymbol{v},(m, \boldsymbol{x}))=f_{\boldsymbol{v}}(\boldsymbol{x}, m)$ which returns $m$ if $\langle\boldsymbol{x}, \boldsymbol{v}\rangle=0$ (i.e,. the two vectors are orthogonal) or $\perp$ otherwise. IPE is a generalization of IdentityBased Encryption [6,9,24] and Anonymous Identity-Based Encryption [1,5], and has been the subject of extensive studies in the last decade.

In FE and IPE, the encryptors and the Central Authority (CA) that generate the tokens are assumed to be honest. Indeed, as noticed by Badrinarayanan et al. in presence of any dishonest party (that is, either the party that generates the token or the party who encrypts the message), the decryption outputs may be inconsistent and this raises serious issues in practical applications (e.g., auditing). For instance, a dishonest authority might be able to generate a faulty token Tok $\boldsymbol{v}_{\boldsymbol{v}}$ for a vector $\boldsymbol{v}$ such that Tok $\boldsymbol{v}$ enables the owner to decrypt a ciphertext for a vector $\boldsymbol{x}$ that is not orthogonal to $\boldsymbol{v}$. Or a dishonest encryptor might generate a faulty ciphertext that decrypts to an incorrect result with an honestly computed token. These issues are particularly severe in the applications to functional commitments that we will see later.

Verifiable Inner Product Encryption (VIPE) overcomes those limitations by adding strong verifiability guarantees to IPE. VIPE is a special case of Verifiable Functional Encryption (VFE), firstly proposed by Badrinarayanan et al. [2] for general functionalities. Informally speaking, in VIPE there are public verification algorithms to verify that the output of the setup, encryption and token generation algorithms are computed honestly. Intuitively, if the master public key MPK and a ciphertext CT pass a public verification test, it means there exists some message $m$ and a unique vector $\boldsymbol{x}$ - up to parallelism - such that for all vectors $\boldsymbol{v}$, if a token Tok $_{\boldsymbol{v}}$ for $\boldsymbol{v}$ is accepted by the verification algorithm then the following holds:

$$
\forall \boldsymbol{v}: \operatorname{Dec}\left(\operatorname{Tok}_{\boldsymbol{v}}, \mathrm{CT}\right)=f_{\boldsymbol{v}}(\boldsymbol{x}, m)
$$

The main component we employ for constructing a VIPE scheme is an IPE scheme. However, it is worth mentioning that most IPE schemes cannot be made verifiable following the general compiler of Badrinarayanan et al. because this compiler requires the IPE scheme to have perfect correctness. We will later discuss in depth why this property is crucial in constructing VIPE.

\subsection{Our Results and Applications}

Our Contribution. In this paper we construct an efficient VIPE scheme from bilinear maps. Towards this goal, we build a perfectly correct IPE scheme that may be of independent interest. To our knowledge, all IPE schemes known in literature do not satisfy perfect correctness. Our perfectly correct IPE scheme is based on standard assumptions over bilinear groups.

We assume the reader to be familiar with the construction of Badrinarayanan et al. [2] that transforms a generic perfectly correct FE scheme to a VFE scheme for the same functionality. They employ four duplicates of the underlying FE scheme 
adding NIWI proofs for verifiabilty with trapdoor statements to ensure privacy. We will use this transform explicitly in Sect. 4. This transform, for the case of the inner-product functionality of [18], requires a perfectly correct IPE scheme and non-interactive witness-indistinguishable (NIWI) proofs for the relations we will define in Sect. 5. Therefore, constructing an efficient VIPE scheme boils down to building an efficient perfectly correct IPE scheme and efficient NIWI proofs for specific relations. The rest of the paper is devoted to achieving these goals.

Motivating Applications. IPE has numerous applications, including Anonymous Identity-Based Encryption [5], Hidden-Vector Encryption [8], and predicate encryption schemes supporting polynomial evaluation [18]. As shown by Badrinarayanan et al. [2], making FE schemes verifiable enables more powerful applications. As an example, in this section we show that VIPE can be used to construct what we call polynomial commitment scheme which corresponds to a functional commitment of Badrinarayanan et al. for the polynomial evaluation predicate. The same construction can easily be adapted to construct functional commitments for the inner-product predicate.

Perfectly Binding Polynomial Commitments. Using a polynomial commitment scheme (see also [17]), Alice may publish a commitment to a polynomial poly $(x)$ with coefficients in $\mathbb{Z}_{p}$. If later Bob wants to know poly $(m)$ for some value $m$, that is the evaluation of the polynomial at some point, he sends $m$ to Alice who replies with the claimed evaluation $y$ and a proof that $y=\operatorname{poly}(m)$. The proof guarantees that the claimed evaluation is consistent with the committed polynomial. We require the scheme to be perfectly binding.

We construct a polynomial commitment scheme for polynomials of degree at most $d$ from a VIPE scheme for vectors of dimension $d+2$ in the following way. Let VIP $=\langle$ VIP.SetUp, VIP.TokGen, VIP.Enc, VIP.Dec $\rangle$ be a VIPE scheme. We define the following algorithms:

- Commitment Phase: To commit to a polynomial poly $(x)=a_{d} x^{d}+a_{d-1} x^{d-1}+$ $\ldots+a_{1} x+a_{0} \in \mathbb{Z}_{p}[X]$, run VIP.SetUp $\left(1^{\lambda}, d+2\right)$ to generate (MPK, MSK), compute the attribute $\boldsymbol{x}:=\left(a_{d}, a_{d-1}, \ldots, a_{1}, a_{0}, 1\right) \in \mathbb{Z}_{p}^{d+2}$ and ciphertext $\mathrm{CT} \rightarrow$ VIP.Enc(MPK, $\boldsymbol{x})$, and output the commitment $:=(\mathrm{MPK}, \mathrm{CT})$.

- Opening phase: In this phase, a party requests a query $(m, y)$ to check if the commitment corresponds to a polynomial poly such that $\operatorname{poly}(m)=$ $y$. The Committer runs the token-generator algorithm of VIP for vector $\boldsymbol{v}:=\left(m^{d}, m^{d-1}, \ldots, m, 1,-y\right)$ and sends Tok $\boldsymbol{v}$ as the opening. Note that $\langle\boldsymbol{x}, \boldsymbol{v}\rangle=a_{d} m^{d}+a_{d-1} m^{d-1}+\ldots+a_{1} m+a_{0}-y=$ poly $(m)-y$, therefore VIP.Dec $\left(\right.$ CT, Tok $\left._{\boldsymbol{v}}\right)=0$ iff poly $(m)=y$.

It is straightforward to see that the above algorithms form a functional commitment (in the sense of [2]) for the polynomial evaluation predicate. We refer the reader to [2] for more details on functional commitments.

\subsection{Technical Overview}

To instantiate the transform of Badrinarayanan et al. we need to build an IPE scheme with perfect correctness. Our starting point to construct a perfectly 
correct IPE scheme is the IPE scheme of Park [22] which only enjoys statistical correctness. The reason for choosing this IPE is that it is conceptually simple and its security is based on standard assumptions over bilinear groups. However, to make the Park's scheme compatible with the Badrinarayanan et al.'s transform we need to solve several technical challenges, in particular:

i. The master public key needs to be verifiable.

ii. The scheme has to satisfy perfect correctness.

This requires substantial modification of all main algorithms: setup, token generation, encryption and decryption.

Verification of Algorithm Outputs. A VIPE scheme requires public verification algorithms that can verify the outputs of the setup, encryption and token generation algorithms, in particular check whether these algorithms were run honestly. In more detail, if any string (master public key, ciphertext or token) passes the corresponding verification algorithm, it means it was a proper output of the corresponding algorithm (setup, encryption or token generation). Each party who runs the setup, encryption or token generation algorithm needs to provide a proof that it executed the algorithm honestly without revealing harmful information about the secret parameters or the randomness used in the algorithm.

Usually non-interactive Zero-Knowledge (NIZK) proofs are used in this context. Unfortunately, NIZK proofs cannot used for verifiable FE as they rely on a trusted CRS (Common Reference String) or random oracles and we aim at perfect verifiability which has to hold despite any collusion and computing power. The transform of Badrinarayanan et al. solves the issue by employing NIWI-proofs in a clever way.

Following the transform of [2], our VIPE consists of four instances of an IPE scheme. In the VIPE's encryption algorithm we first run the IPE's encryption algorithm four times to generate four ciphertexts and then we prove that all these four ciphertexts are the encryption of the same message or that some other trapdoor predicate is satisfied (the latter is needed for message indistinguishability and will be detailed later).

For the sake of argument, let us assume the VIPE scheme consists only of two (instead of four) parallel perfectly correct IPE scheme instantiations IP and IP. The master public key of the Park's scheme [22] contains a component $\Lambda=e\left(g, g^{\prime}\right)$ in which $g$ is public but $g^{\prime}$ needs to be kept secret. An honestly computed ciphertext CT in IP includes $\mathrm{ct}_{1}=g^{-s}$ and $\mathrm{ct}_{7}=\Lambda^{-s} \cdot m$ among its components (we here ignore the other components). We first provide proof that CT (resp. CT in IP) is well-formed. Then we need to prove that the two ciphertexts are both encryptions of the same message $M$ (i.e., $m=m \hat{=} M$ ). We reduce the problem to proving that the following property holds:

$$
\frac{\mathrm{ct}_{7}}{\hat{\mathrm{ct}} \mathrm{t}_{7}}=\frac{e\left(g, g^{\prime}\right)^{-s} \cdot m}{e\left(\hat{g}, \hat{g}^{\prime}\right)^{-\hat{s}} \cdot \hat{m}}=\frac{e\left(\hat{\mathrm{ct}}_{1}, \hat{g}^{\prime}\right)}{e\left(\mathrm{ct}_{1}, g^{\prime}\right)}=\frac{e\left(\hat{g}^{\hat{s}}, \hat{g}^{\prime}\right)}{e\left(g^{s}, g^{\prime}\right)}
$$

However, since $g^{\prime}$ and $\hat{g}^{\prime}$ are not public, the party who runs the encryption algorithm would be unable to prove this property. We solve this issue in the 
following way: We add to the master public key of IP two elements $g_{1}, g_{2}$ (and $\hat{g}_{1}, \hat{g}_{2}$ for $\left.\mathbf{I P}\right)$ satisfying $\Lambda=e\left(g, g^{\prime}\right)=e\left(g_{1}, g_{2}\right), \hat{\Lambda}=e\left(\hat{g}, \hat{g}^{\prime}\right)=e\left(\hat{g}_{1}, \hat{g}_{2}\right)$. Then, we add the following equations for the new secret variables $\mathcal{X}_{3}=g_{1}^{s}, \hat{\mathcal{X}}_{3}=\hat{g}_{1}^{\hat{s}}$ :

$$
\mathrm{ct}_{7}^{-1} \cdot \hat{\mathrm{ct}}_{7}=e\left(\mathcal{X}_{3}, g_{2}\right) \cdot e\left(\hat{\mathcal{X}}_{3}, \hat{g}_{2}\right)^{-1}, e\left(g, \mathcal{X}_{3}\right)=e\left(\mathrm{ct}_{1}, g_{1}\right), e\left(\hat{g}, \hat{\mathcal{X}}_{3}\right)=e\left(\hat{\mathrm{ct}}_{1}, \hat{g}_{1}\right)
$$

It is easy to see that these equations are satisfied iff $m=\hat{m}$, and now they can be proven by the encryptor. Having modified Park's scheme, we thus have to prove that the modified scheme is IND-secure. This is done in Sect. 3.1 in which we reduce the IND-Security of the scheme to the Decision Linear assumption.

Achieving Perfect Correctness. For the Badrinarayanan et al.'s transform to work, it is crucial that the underlying IPE scheme have perfect correctness. If the IPE scheme had a negligible probability of decryption error rather than perfect correctness, then dishonest parties might collude with each other so that invalid results would be accepted by the verification algorithms. Contrast this with the aforementioned functional commitments. In the latter primitive, the committer is the same party who generates the ciphertext (the commitment) and the token (the decommitment) and thus might profit from a negligible space of decryption error to prove false assertions on its committed value. To our knowledge, all IPE schemes ${ }^{1}$ known in the literature have a negligible probability of error which makes cheating possible and so not directly usable to construct verifiable functional encryption and functional commitments for the IPE functionality.

In more detail, in most pairing-based IPE schemes the encryption and decryption algorithms work as follows:

$$
\operatorname{Enc}(\mathrm{MPK}, \boldsymbol{x}, \mathbf{m}) \rightarrow \mathrm{CT}, \operatorname{Dec}\left(\mathrm{Tok}_{\boldsymbol{v}}, \mathrm{CT}\right) \rightarrow m^{*}=m \cdot(\mathrm{r})^{\langle\boldsymbol{x}, \boldsymbol{v}\rangle},
$$

in which $r$ is some random value that depends on the randomness used by the token generator and encryption algorithms. Thus, even in case of honest parties, there is a negligible probability that $\mathrm{r}=1$ and so, even if $\langle\boldsymbol{x}, \boldsymbol{v}\rangle \neq 0$, the decryption algorithm may output a valid message $\mathbf{m}$ instead of $\perp$.

In case of dishonest parties, it may happen that two parties (the encryptor and the token generator) collude with each other to create randomness such that $r$ equals 1 . In this case, the parties would be able to provide valid proofs of the fact that they followed the protocol correctly and invalid results would pass the verification algorithms. A similar problem also appears in the context of MPC in the head [16], where the soundness of the ZK protocol built from MPC strongly relies on the perfect correctness of the underlying MPC. To cope with statistical correctness in MPC in the head, a coin tossing protocol can be employed, while in a completely non-interactive scenario like ours this is more challenging. Hence, to obtain a VIPE scheme it is crucial to construct an IPE scheme satisfying perfect correctness.

Recall that the decryption algorithm in the IPE scheme of Park [22] works as follows:

$$
\operatorname{Dec}\left(\operatorname{Tok}_{\boldsymbol{v}}, \mathrm{CT}=\operatorname{Enc}(\boldsymbol{x}, m)\right) \longrightarrow m^{*}=m \cdot \mathbf{e}(g, h)^{\left(\lambda_{1} s_{3}+\lambda_{2} s_{4}\right)\langle\boldsymbol{x}, \boldsymbol{v}\rangle}
$$

\footnotetext{
${ }^{1}$ Recall that we refer to the IPE functionality of Katz, Sahai and Waters [18].
} 
in which $\lambda_{1}, \lambda_{2}$ are random values used in the token generation algorithm and $s_{3}, s_{4}$ are random values used in the encryption algorithm. To decide whether to accept the output of the decryption or not, the first attempt would be the following. Generate two ciphertexts ct, $\mathrm{ct}^{\prime}$ with two independent random values $\left\{s_{i}\right\},\left\{s_{i}^{\prime}\right\}$, decrypt both ct and $\mathrm{ct}^{\prime}$ to get $M$ and $M^{\prime}$ and if $M=M^{\prime}$ accept the result, or output $\perp$ otherwise. In more detail:

$$
M=m \cdot e(h, g)^{\left(\lambda_{1} s_{3}+s_{4} \lambda_{2}\right)\langle\boldsymbol{x}, \boldsymbol{v}\rangle}, M^{\prime}=m \cdot e(h, g)^{\left(\lambda_{1} s_{3}^{\prime}+s_{4}^{\prime} \lambda_{2}\right)\langle\boldsymbol{x}, \boldsymbol{v}\rangle}
$$

However, in case $\langle\boldsymbol{x}, \boldsymbol{v}\rangle \neq 0$ there is non-zero probability for which:

$$
\lambda_{1} s_{3}+s_{4} \lambda_{2}=\lambda_{1} s_{3}^{\prime}+\lambda_{2} s_{4}^{\prime} \neq 0 \Rightarrow M=M^{\prime} \neq m
$$

To avoid this issue, we choose the random values in such a way that the above equality can never occur. To do so, in the encryption algorithm we choose nonzero random values $s_{1}, \ldots, s_{4}$ and $s_{1}^{\prime}, \ldots, s_{4}^{\prime}$ such that $s_{3} \neq s_{3}^{\prime}$, and $s_{4}=s_{4}^{\prime}$. In this case, we have:

$$
\lambda_{1} s_{3}+s_{4} \lambda_{2}=\lambda_{1} s_{3}^{\prime}+\lambda_{2} s_{4} \Rightarrow \lambda_{1}\left(s_{3}-s_{3}^{\prime}\right)=0 \Rightarrow\left(\lambda_{1}=0\right) \vee\left(s_{3}=s_{3}^{\prime}\right)
$$

Based on the way $\lambda_{1}, s_{3}, s_{3}^{\prime}$ have been chosen, neither $\left(\lambda_{1}=0\right)$ nor $\left(s_{3}=s_{3}^{\prime}\right)$ may happen, hence the decryption algorithm outputs $m$ if and only if $\langle\boldsymbol{x}, \boldsymbol{v}\rangle=0$. The resulting IPE scheme satisfies perfect correctness as wished and we prove that it is still selectively indistinguishability-secure under the DLin Assumption. When constructing a VIPE scheme from such IPE scheme, these additional constraints in the encryption and token generation procedures will correspond to more constraints in the proofs of correct encryption and token generation.

Furthermore, an additional challenge we will have to address is that some of the proofs in the Badrinarayanan et al. transform are for relations that consist of a generalized form of disjunction and thus standard techniques to implement disjunctions for GS proofs cannot be directly applied, see Sect.5.1.

\subsection{Related Work and Comparison}

Verifiable functional encryption has been introduced by Badrinarayanan et al. [2], who provide a construction for general functionalities.

Recently, [3] introduced a new FE scheme that supports an extension of the inner-product functionality. The scheme is perfectly correct assuming the message space to be short. However, notice that when employing the scheme in order to construct an IPE scheme (according to the functionality of Katz, Sahai and Waters [18]) the perfect correctness is lost. In essence, the IPE constructed from the scheme in [3] would encrypt some additional random value $r$ so that the decryption would return the value $m+r \cdot\langle\boldsymbol{x}, \boldsymbol{v}\rangle$. In this way, if the vectors $\boldsymbol{x}$ and $\boldsymbol{v}$ are orthogonal then the payload message $m$ is obtained, otherwise a random value is returned.

As corollary of our VIPE, we obtain functional commitments (in the sense of [2]) for the polynomial evaluation and inner-product predicate. A similar form 
of commitments has been proposed by Libert et al. [20] but differs from ours in different aspects. In the Libert et al.'s scheme, the decommitter reveals the evaluations of the inner-product of the committed vector with any vector of its choice, whereas in ours just the binary value of the inner-product predicate (i.e whether the two vectors are orthogonal or not) is leaked. Our functional commitments are perfectly binding rather than computational binding as in Libert et al. Moreover, ours are not based on any trust assumption, whereas in [20] the generator of the public-key can completely break the binding property.

Tang and Ji [26] constructed an Attribute-based Encryption scheme that enjoys a weaker form of verifiability limited to the secret keys.

Roadmap. In Sect. 2 we provide the building blocks and the basic terminology used in this paper. In Sect. 3 we construct our perfectly correct IPE scheme and prove its security based on the Decisional Bilinear Diffie-Hellman and DLin assumptions. In Sect. 4 we define VIPE and present one candidate construction built on perfectly correct IPE and the NIWI proofs of Sect. 5 .

\section{Preliminaries}

Notation. Throughout the paper, we use $\lambda \in \mathbb{N}$ as a security parameter. For any integer $n>0$, we denote by $[n]$ the set $\{1, \ldots, n\}$. PPT stands for probabilistic polynomial time algorithm and $\operatorname{negl}(\lambda)$ denotes a negligible function in $\lambda$.

\section{$2.1 \quad$ Building Blocks}

Definition 1 (Bilinear group [6]). A bilinear group consists of a pair of groups $\mathbb{G}$ and $\mathbb{G}_{T}$ of prime order $p$ with a map e : $\mathbb{G} \times \mathbb{G} \rightarrow \mathbb{G}_{T}$ satisfying:

1. Bilinearity: for all $a, b \in \mathbf{Z}, e\left(g^{a}, g^{b}\right)=e(g, g)^{a b}$ for any $g \in \mathbb{G}$.

2. Non-degeneracy: $e(g, g) \neq 1_{\mathbb{G}_{T}}$ for any $g \in \mathbb{G}$.

3. Efficiency: there exists an efficient algorithm to compute the map.

Definition 2 (NIWI). A non-interactive witness indistinguishable proof system $(N I W I)$ is a pair of PPT algorithms $\langle\mathcal{P}, \mathcal{V}\rangle$ for a $N P$-relation $R_{L}$ satisfying the following properties:

1. Completeness: for all $(x, w) \in R_{L}, \operatorname{Pr}[\mathcal{V}(x, \pi)=1 \mid \pi \longleftarrow \mathcal{P}(x, w)]=1$.

2. Perfect soundness: for every $x \notin L$ and $\pi \in\{0,1\}^{*}, \operatorname{Pr}[\mathcal{V}(x, \pi)=1]=0$.

3. Witness indistinguishability: for any sequence $\left\{\left(x_{n}, w_{1, n}, w_{2, n}\right)\right\}_{n \in \mathbb{N}}$, which $x_{n} \in\{0,1\}^{n}, w_{1, n}, w_{2, n} \in R_{L}\left(x_{n}\right)$, the following holds:

$n \in \mathbb{N}:\left\{\pi_{1, n} \mid \pi_{1, n} \leftarrow \mathcal{P}\left(x_{n}, w_{1, n}\right)\right\}_{n} \approx_{c}\left\{\pi_{2, n} \mid \pi_{2, n} \leftarrow \mathcal{P}\left(x_{n}, w_{2, n}\right)\right\}_{n}$.

Groth and Sahai (GS) [14] provide NIWI systems for the satisfiability of what they call "Pairing Products Equations" that can be used to instantiate the relations needed in our VIPE construction (cf. Construction 7). Using the techniques of [13], such proofs may be made perfectly sound. 
IPE Scheme: For any $n>0$, let $\Sigma_{n}$ be a set of vectors of length $n$ defined over some field and let $\mathcal{M}$ be a message space. For any vector $\boldsymbol{v} \in \Sigma_{n}$, the function $f_{v}: \Sigma_{n} \times \mathcal{M} \rightarrow \mathcal{M} \cup\{\perp\}$ is

$$
f_{\boldsymbol{v}}(\boldsymbol{x}, m)=\left\{\begin{array}{l}
m \text { If }\langle\boldsymbol{x}, \boldsymbol{v}\rangle=0 \\
\perp \text { If }\langle\boldsymbol{x}, \boldsymbol{v}\rangle \neq 0
\end{array} .\right.
$$

Both $\mathcal{M}, n$ and the field size can depend on the security parameter $\lambda$ but for simplicity hereafter we will skip this detail. IPE can be seen as a FE scheme for the previous functionality. More concretely, an IPE scheme is defined as follows.

Definition 3 (IPE Scheme). An IPE scheme IP for a message space $\mathcal{M}$ and for a family of sets $\Sigma=\left\{\Sigma_{n}\right\}_{n>0}$ consisting of sets of vectors of length $n$ over some field is a tuple of four PPT algorithms IP $=$ IIP.SetUp, IP.TokGen, IP.Enc, IP.Dec $\}$ with the following syntax and satisfying the correctness property below.

- IP.SetUp $\left(1^{\lambda}, n\right) \longrightarrow(\mathrm{MPK}, \mathrm{MSK})$ : the setup algorithm, on input the security parameter $\lambda$ and the vector length $n$, generates master public key MPK and master secret key MSK for that parameter.

- IP.TokGen(MPK, MSK, $\boldsymbol{v}) \longrightarrow$ Tok $\boldsymbol{v}$ : on input master keys and vector $\boldsymbol{v} \in \Sigma_{n}$, the token generation algorithm generates the token Tok .

- IP.Enc(MPK, $\vec{x}, m) \longrightarrow \mathrm{CT}$ : the encryption algorithm encrypts message $m \in$ $\mathcal{M}$ and vector $\boldsymbol{x} \in \Sigma_{n}$ under the master public key.

- IP.Dec(MPK, Tok $v, C T) \longrightarrow m^{\prime} \in \mathcal{M} \cup\{\perp\}$.

- Perfect correctness: IP is perfectly correct if for all $\lambda, n>0, \boldsymbol{x}, \boldsymbol{v} \in \Sigma_{n}$ and all $m \in \mathcal{M}$ the following holds:

$$
\operatorname{Pr}\left[\begin{array}{ll}
\text { IP.Dec }\left(\mathrm{MPK}, \mathrm{Tok}_{\boldsymbol{v}}, \mathrm{CT}\right) & (\mathrm{MPK}, \mathrm{MSK}) \longleftarrow \text { IP.SetUp }\left(1^{\lambda}, n\right), \\
=f_{\boldsymbol{v}}(\boldsymbol{x}, m) & \text { Tok }_{\boldsymbol{v}} \longleftarrow \mathrm{IP} . T o k G e n(M P K, \mathrm{MSK}, \boldsymbol{v}), \\
& \text { CT } \longleftarrow \text { IP.Enc }(\mathrm{MPK}, \boldsymbol{x}, m)
\end{array}\right]=1
$$

Security. To model security we adopt the indistinguishability-based (IND) notion of security [8], in particular selective security [4]. Boneh, Sahai, and Waters [7] showed deficiencies of this notion in general and impossibility results for the more general notion of simulation-based security; see also [7,10,11,15] for general techniques to overcome the known impossibility results in different settings. Nonetheless, to our knowledge no practical attacks are known for natural schemes. Selective security is sufficient for CCA-security [4] and for our application of verifiable polynomial commitments of Sect.1.1.

The selectively indistinguishability-based notion of security for an IPE scheme over the vector space $\Sigma$ and message space $\mathcal{M}$ is formalized by means of the game $\operatorname{IND}^{\mathcal{A}, \mathcal{C}, \lambda, n}$ in Fig. 1, between an adversary $\mathcal{A}$ and a challenger $\mathcal{C}$ (defined in the game) parameterized by security parameter $\lambda$ and dimension $n$. The advantage of $\mathcal{A}$ in this game is $\operatorname{Adv}_{\mathrm{IP}, \lambda, n}(\mathcal{A})=\left|\operatorname{Pr}\left[\operatorname{IND}^{\mathcal{A}, \mathrm{IP}, \lambda, n}=1\right]-\frac{1}{2}\right|$.

Definition 4. An IPE scheme IP is selectively-indistinguishable secure (INDSecure) if for all $n>0$ and all PPT adversaries $\mathcal{A}, \operatorname{Adv}_{\mathrm{IP}, \lambda, n}(\mathcal{A})$ is a negligible function of $\lambda$. 
- Selective Challenge Phase. $\mathcal{A}\left(1^{\lambda}, n\right) \longrightarrow \boldsymbol{x}_{0}, \boldsymbol{x}_{1} \in \Sigma_{n}$. Then $\mathcal{A}$ sends these two vectors to the challenger.

- Setup Phase. The challenger $\mathcal{C}$ generates the pair (MSK, MPK) by invoking the setup algorithm on input $\left(1^{\lambda}, n\right)$. Then $\mathcal{C}$ sends MPK to $\mathcal{A}$.

- Query Phase 1. $\mathcal{A}$ asks for the token for a vector $\boldsymbol{v}_{\boldsymbol{i}} \in \Sigma_{n}$.

- Challenge Phase. $\mathcal{A}$ sends to the challenger two messages $m_{0}, m_{1} \in \mathcal{M}$ of the same length.

$-\mathcal{C}$ flips a coin to generate random bit $b$ and send CT $=\operatorname{Enc}\left(\mathrm{MPK}, \boldsymbol{x}_{\boldsymbol{b}}, m_{b}\right)$.

- Query Phase 2. Query Phase 2: same as Query Phase 1.

- Output Phase. $\mathcal{A}$ outputs a bit $b^{\prime}$.

- Winning Condition. $\mathcal{A}$ wins the game if $b^{\prime}=b$ and the following condition is met. It is required that if $m_{0} \neq m_{1},\left\langle\boldsymbol{x}_{0}, \boldsymbol{v}_{i}\right\rangle,\left\langle\boldsymbol{x}_{1}, \boldsymbol{v}_{i}\right\rangle \neq 0$ for all the vectors $\boldsymbol{v}_{i}$ queried in both query phase 1 and 2 , or $\left\langle\boldsymbol{v}_{i}, \boldsymbol{x}_{0}\right\rangle=0$ iff $\left\langle\boldsymbol{v}_{i}, \boldsymbol{x}_{1}\right\rangle=0$ otherwise. If the winning condition is satisfied the output of the game is 1 or 0 otherwise.

Fig. 1. Security Game IND ${ }^{\mathcal{A}, I P, \lambda, n}$

\subsection{Hardness Assumptions}

We conjecture that the following problems hold relative to some bilinear group generator GroupGen $\left(1^{\lambda}\right) \rightarrow\left(p, \mathbb{G}, \mathbb{G}_{T}, e\right)$ that takes security parameter $\lambda$ as input and outputs $\lambda$-bit prime $p$, the descriptions of two groups $\mathbb{G}$ and $\mathbb{G}_{T}$ of order $p$ and a bilinear map $e: \mathbb{G} \times \mathbb{G} \rightarrow \mathbb{G}_{T}$.

Assumption 1. The Decisional Bilinear Diffie-Hellman assumption (DBDH) in bilinear groups $\left(p, \mathbb{G}, \mathbb{G}_{T}, e\right)$ states the hardness for PPT adversaries of solving the following problem. On input $\left(g, g^{\alpha}, g^{\beta}, g^{\gamma}, Z\right) \in \mathbb{G}^{4} \times \mathbb{G}_{T}$, decide whether $Z=e\left(g, g^{\alpha \beta \gamma}\right)$ or $Z$ is a random element in $\mathbb{G}_{T}$.

Assumption 2. The Decisional Linear assumption (DLin) in a bilinear group $\left(p, \mathbb{G}, \mathbb{G}_{T}, e\right)$ states the hardness for PPT adversaries of solving the following problem. On input $\left(g, g^{\alpha}, g^{\beta}, g^{\alpha \tau}, g^{\beta \eta}, Z\right) \in \mathbb{G}^{6}$, decide whether $Z=g^{\eta+\tau}$ or $a$ random element in $\mathbb{G}$.

In this paper we use the following equivalent formulation of DLin given in [22]: on input $\left(g, g^{\alpha}, g^{\beta}, g^{\tau}, g^{\alpha \eta}, Z\right) \in \mathbb{G}^{6}$ decide whether $Z=g^{\beta(\eta+\tau)}$ or a random element.

Note that DLin is stronger than DBDH. In the rest of this paper we assume the existence of a bilinear group generator GroupGen such that DLin (and thus DBDH) holds relative to it.

\section{Our Perfectly Correct Inner-Product Encryption}

In this section we construct our perfectly correct IPE, the key ingredient for building verifiable inner-product encryption (see Sect.4). 
Let $\operatorname{Group} \operatorname{Gen}\left(1^{\lambda}\right) \longrightarrow\left(p, \mathbb{G}, \mathbb{G}_{T}, e\right)$ be a bilinear group generator, and $n \in \mathbb{N}$ be the vector length. We construct a perfectly correct IPE scheme IP $=\langle$ IP.SetUp, IP.Enc, IP.TokGen, IP.Dec $\rangle$ for the set $\mathbb{Z}_{p}^{n}$ of vectors of length $n$ over $\mathbb{Z}_{p}$ and for message space $\mathcal{M}=\mathbb{G}_{T}$.

Construction 1 [Our perfectly correct IPE scheme IP]

- IP.SetUp $\left(1^{\lambda}, n\right) \longrightarrow($ MSK, MPK):

For security parameter $\lambda, i \in[n]$ and $b \in[2]$, compute what follows:

1. Run GroupGen $\left(1^{\lambda}\right)$ (cf. Sect. 2.2) to generate a tuple $\left\langle p, \mathbb{G}, \mathbb{G}_{T}, e\right\rangle$.

2. Pick $g, g^{\prime} \leftarrow \mathbb{G}$ and $\delta_{1}, \theta_{1}, \delta_{2}, \theta_{2}, w_{1, i}, t_{1, i}, f_{b, i}, h_{b, i}, k \leftarrow \mathbb{Z}_{p}^{*}$.

3. Pick $\Omega \leftarrow \mathbb{Z}_{p}$ and compute $\left\{w_{2, i}, t_{2, i}\right\}_{i \in[n]}$ such that:

$$
\Omega=\delta_{1} w_{2, i}-\delta_{2} w_{1, i}=\theta_{1} t_{2, i}-\theta_{2} t_{1, i} .
$$

4. For $i \in[n], b[2]$ set:

$$
\begin{array}{lllll}
W_{b, i}=g^{w_{b, i}}, & F_{b, i}=g^{f_{b, i}}, & K_{1}=g^{k}, & U_{b}=g^{\delta_{b}}, & h=g^{\Omega}, \\
T_{b, i}=g^{t_{b, i}}, & H_{b, i}=g^{h_{b, i}}, & K_{2}=g^{\prime \frac{1}{k}}, & V_{b}=g^{\theta_{b}}, & \Lambda=\mathbf{e}\left(g, g^{\prime}\right) .
\end{array}
$$

5. Set:

$$
\begin{aligned}
\text { MPK }= & {\left[\left(p, \mathbb{G}, \mathbb{G}_{t}, e\right),\left(g, h,\left\{W_{b, i}, F_{b, i}, T_{b, i}, H_{b, i}, U_{b}, V_{b}\right\}_{b \in[2], i \in[n]},\right.\right.} \\
& \left.\left.K_{1}, K_{2}, \Lambda\right) \in \mathbb{G}^{8 n+8} \times \mathbb{G}_{T}\right], \\
\mathrm{MSK}= & \left(\left\{w_{b, i}, f_{b, i}, t_{b, i}, h_{b, i}, \delta_{b}, \theta_{b}\right\}_{b \in[2], i \in[n]}, g^{\prime}\right) \in \mathbb{Z}_{p}^{8 n+4} \times \mathbb{G} .
\end{aligned}
$$

6. Return (MPK, MSK).

- IP.Enc(MPK, $\boldsymbol{x}, m) \longrightarrow$ CT:

1. For $\boldsymbol{x}=\left(x_{1}, \ldots, x_{n}\right) \in \mathbb{Z}_{p}^{n}$ and a message $m \in \mathbb{G}_{T}$, pick random elements $s_{1}, \ldots s_{4}, s_{1}^{\prime}, \ldots, s_{3}^{\prime} \leftarrow \mathbb{Z}_{p}^{*}$ such that $s_{3} \neq s_{3}^{\prime}$ and compute what follows:

$$
\begin{aligned}
& \mathrm{ct}_{1}=g^{s_{2}}, \mathrm{ct}_{2}=h^{s_{1}}, \\
& \left\{\begin{array}{l}
\mathrm{ct}_{3, i}=W_{1, i}^{s_{1}} \cdot F_{1, i}^{s_{2}} \cdot U_{1}^{x_{i} s_{3}}, \mathrm{ct}_{4, i}=W_{2, i}^{s_{1}} \cdot F_{2, i}^{s_{2}} \cdot U_{2}^{x_{i} s_{3}} \\
\mathrm{ct}_{5, i}=T_{1, i}^{s_{1}} \cdot H_{1, i}^{s_{2}} \cdot V_{1}^{x_{i} s_{4}}, \mathrm{ct}_{6, i}=T_{2, i}^{s_{1}} \cdot H_{2, i}^{s_{2}} \cdot V_{2}^{x_{i} s_{4}}
\end{array}\right\}_{i \in[n]}, \\
& \mathrm{ct}_{7}=\mathbf{e}\left(g^{s_{3}}, g^{s_{4}}\right), \mathrm{ct}_{8}=\Lambda^{-s_{2}} \cdot m . \\
& \mathrm{ct}_{1}^{\prime}=g^{s_{2}^{\prime}}, \mathrm{ct}_{2}^{\prime}=h^{s_{1}^{\prime}}, \\
& \left\{\begin{array}{l}
\mathrm{ct}_{3, i}^{\prime}=W_{1, i}^{s_{1}^{\prime}} \cdot F_{1, i}^{s_{2}^{\prime}} \cdot U_{1}^{x_{i} s_{3}^{\prime}}, \mathrm{ct}_{4, i}^{\prime}=W_{2, i}^{s_{1}^{\prime}} \cdot F_{2, i}^{s_{2}^{\prime}} \cdot U_{2}^{x_{i} s_{3}^{\prime}} \\
\mathrm{ct}_{5, i}^{\prime}=T_{1, i}^{s_{1}^{\prime}} \cdot H_{1, i}^{s_{2}^{\prime}} \cdot V_{1}^{x_{i} s_{4}}, \mathrm{ct}_{6, i}^{\prime}=T_{2, i}^{s_{1}^{\prime}} \cdot H_{2, i}^{s_{2}^{\prime}} \cdot V_{2}^{x_{i} s_{4}}
\end{array}\right\}, \\
& \mathrm{ct}_{7}^{\prime}=\mathbf{e}\left(g^{s_{3}^{\prime}}, g^{s_{4}}\right), \mathrm{ct}_{8}^{\prime}=\Lambda^{-s_{2}^{\prime}} \cdot m .
\end{aligned}
$$

2. Set:

$$
\begin{aligned}
& \mathrm{ct}=\left(\mathrm{ct}_{1}, \mathrm{ct}_{2},\left\{\begin{array}{l}
\mathrm{ct}_{3, i}, \mathrm{ct}_{4, i} \\
\mathrm{ct}_{5, i}, \mathrm{ct}_{6, i}
\end{array}\right\}, \mathrm{ct}_{7}, \mathrm{ct}_{8}\right), \\
& \mathrm{ct}^{\prime}=\left(\mathrm{ct}_{1}^{\prime}, \mathrm{ct}_{2}^{\prime},\left\{\begin{array}{l}
\mathrm{ct}_{3, i}^{\prime}, \mathrm{ct}_{4, i}^{\prime} \\
\mathrm{ct}_{5, i}^{\prime}, \mathrm{ct}_{6, i}^{\prime}
\end{array}\right\}, \mathrm{ct}_{7}^{\prime}, \mathrm{ct}_{8}^{\prime}\right) .
\end{aligned}
$$


3. Output $\mathrm{CT}=\left(\mathrm{ct}, \mathrm{ct}^{\prime}\right)$.

- IP.TokGen(MSK, $v) \longrightarrow$ Tok $_{v}$ :

1. Pick $\lambda_{1}, \lambda_{2} \leftarrow \mathbb{Z}_{p}^{*}$ and for any $i \in[n]$ pick $\left\{r_{i}\right\},\left\{\Phi_{i}\right\} \leftarrow \mathbb{Z}_{p}^{*}$.

2. Set Tok $_{\boldsymbol{v}}=\left(K_{A}, K_{B},\left\{\begin{array}{l}K_{3, i}, K_{4, i} \\ K_{5, i}, K_{6, i}\end{array}\right\}\right)$ ) as follows and return Tok . $_{i \in[n]}$

$$
\begin{array}{lrl}
K_{A}=g^{\prime} \cdot \prod_{i=1}^{n} K_{3, i}^{-f_{1, i}} \cdot K_{4, i}^{-f_{2, i}} \cdot K_{5, i}^{-h_{1, i}} \cdot K_{6, i}^{-h_{2, i}}, & K_{B}=\prod_{i=1}^{n} g^{-\left(r_{i}+\Phi_{i}\right)} . \\
K_{3, i}=g^{-\delta_{2} r_{i}} \cdot g^{\lambda_{1} v_{i} w_{2, i},} & K_{4, i}=g^{\delta_{1} r_{i}} \cdot g^{-\lambda_{1} v_{i} w_{1, i}} . \\
K_{5, i}=g^{-\theta_{2} \Phi_{i}} \cdot g^{\lambda_{2} v_{i} t_{2, i}}, & K_{6, i}=g^{\theta 1 \Phi_{i}} \cdot g^{-\lambda_{2} v_{i} t_{1, i}} .
\end{array}
$$

- IP.Dec(CT, Tok $\left._{v}\right)$ :

Let $\mathrm{CT}=\left(\mathrm{ct}, \mathrm{ct}^{\prime}\right)$, such that $\mathrm{ct}=\left(\mathrm{ct}_{1}, \mathrm{ct}_{2},\left\{\mathrm{ct}_{3, i}, \mathrm{ct}_{4, i}, \mathrm{ct}_{5, i}, \mathrm{ct}_{6, i}\right\}, \mathrm{ct}_{7}, \mathrm{ct}_{8}\right)$, $\mathrm{ct}^{\prime}=\left(\mathrm{ct}_{1}^{\prime}, \mathrm{ct}_{2}^{\prime},\left\{\mathrm{ct}_{3, i}^{\prime}, \mathrm{ct}_{4, i}^{\prime}, \mathrm{ct}_{5, i}^{\prime}, \mathrm{ct}_{6, i}^{\prime}\right\}, \mathrm{ct}_{7}, \mathrm{ct}_{8}\right)$

1. If $\mathrm{ct}_{7}=\mathrm{ct}_{7}^{\prime}$ output $\perp$ and stop, otherwise go to the next step.

2. Compute:

$$
\begin{aligned}
\Upsilon= & \mathrm{ct}_{8} \cdot \mathbf{e}\left(\mathrm{ct}_{1}, K_{A}\right) \cdot \mathbf{e}\left(\mathrm{ct}_{2}, K_{B}\right) \cdot \\
& \prod_{i=1}^{n} \mathbf{e}\left(\mathrm{ct}_{3, i}, K_{3, i}\right) \cdot \mathbf{e}\left(\mathrm{ct}_{4, i}, K_{4, i}\right) \cdot \mathbf{e}\left(\mathrm{ct}_{5, i}, K_{5, i}\right) \cdot \mathbf{e}\left(\mathrm{ct}_{6, i}, K_{6, i}\right) . \\
\Upsilon^{\prime}= & \mathrm{ct}_{8}^{\prime} \cdot \mathbf{e}\left(\mathrm{ct}_{1}^{\prime}, K_{A}\right) \cdot \mathbf{e}\left(\mathrm{ct}_{2}^{\prime}, K_{B}\right) . \\
& \prod_{i=1}^{n} \mathbf{e}\left(\mathrm{ct}_{3, i}^{\prime}, K_{3, i}\right) \cdot \mathbf{e}\left(\mathrm{ct}_{4, i}^{\prime}, K_{4, i}\right) \cdot \mathbf{e}\left(\mathrm{ct}_{5, i}^{\prime}, K_{5, i}\right) \cdot \mathbf{e}\left(\mathrm{ct}_{6, i}^{\prime}, K_{6, i}\right) .
\end{aligned}
$$

3. If $\Upsilon=\Upsilon^{\prime}$ output $\Upsilon$ otherwise output $\perp$.

Perfect Correctness: We now show that an honestly generated ciphertext decrypts correctly with probability 1 . Since $F_{1, i}^{-s_{2}} \cdot \mathrm{ct}_{3, i}=W_{1, i}^{s_{1}} \cdot U_{1}^{s_{3} x_{i}}$, we get

$$
\begin{aligned}
& \mathbf{e}\left(F_{1, i}^{-s_{2}} \cdot \mathrm{ct}_{3, i}, K_{3, i}\right)=\mathbf{e}(g, g)^{s_{1} \lambda_{1} v_{i} w_{1, i} w_{2, i}-s_{3} x_{i} \delta_{1} \delta_{2}} \cdot \mathbf{e}(g, g)^{-s_{1} r_{i} \delta_{2} w_{1, i}+s_{3} \lambda_{1} v_{i} \delta_{1} w_{2, i}} \\
& \mathbf{e}\left(F_{2, i}^{-s_{2}} \cdot \mathrm{ct}_{4, i}, K_{4, i}\right)=\mathbf{e}(g, g)^{-s_{1} \lambda_{1} v_{i} w_{1, i} w_{2, i}+s_{3} x_{i} \delta_{1} \delta_{2}} \cdot \mathbf{e}(g, g)^{s_{1} r_{i} \delta_{1} w_{2, i}-s_{3} \lambda_{1} v_{i} \delta_{2} w_{1, i}}
\end{aligned}
$$

We then get

$$
\begin{aligned}
& \mathbf{e}\left(F_{1, i}^{-s_{2}} \cdot \mathrm{ct}_{3, i}, K_{3, i}\right) \cdot \mathbf{e}\left(F_{2, i}^{-s_{2}} \cdot \mathrm{ct}_{4, i}, K_{4, i}\right)= \\
& \left(\mathbf{e}\left(g^{s_{1}}, g^{r_{i}}\right) \cdot \mathbf{e}\left(g^{x_{i} s_{3}}, g^{\lambda_{1} v_{i}}\right)\right)^{\delta_{1} w_{2, i}-\delta_{2} w_{1, i}}= \\
& \quad \mathbf{e}\left(h^{s_{1}}, g^{r_{i}}\right) \cdot \mathbf{e}\left(h^{s_{3} \lambda_{1}}, g^{x_{i} v_{i}}\right)=\mathbf{e}\left(\mathrm{ct}_{2}, g^{r_{i}}\right) \cdot \mathbf{e}\left(h^{\lambda_{1} s_{3}}, g^{x_{i} v_{i}}\right)
\end{aligned}
$$

The same computation gives us

$$
\mathbf{e}\left(H_{1, i}^{-s_{2}} \cdot \mathrm{ct}_{5, i}, K_{5, i}\right) \cdot \mathbf{e}\left(H_{2, i}^{-s_{2}} \cdot \mathrm{ct}_{6, i}, K_{6, i}\right)=\mathbf{e}\left(\mathrm{ct}_{2}, g^{\Phi_{i}}\right) \cdot \mathbf{e}\left(h^{\lambda_{2} s_{4}}, g^{x_{i} v_{i}}\right)
$$


As a conclusion we have the following:

$$
\begin{aligned}
& \mathbf{e}\left(\mathrm{ct}_{1}, K_{A}\right) \cdot \prod_{i=1}^{n} \mathbf{e}\left(\mathrm{ct}_{3, i}, K_{3, i}\right) \cdot \mathbf{e}\left(\mathrm{ct}_{4, i}, K_{4, i}\right) \cdot \mathbf{e}\left(\mathrm{ct}_{5, i}, K_{5, i}\right) \cdot \mathbf{e}\left(\mathrm{ct}_{6, i}, K_{6, i}\right)= \\
& =\Lambda^{s_{2}} \prod_{i=1}^{n} \mathbf{e}\left(F_{1, i}^{-s_{2}}, K_{3, i}\right) \mathbf{e}\left(F_{1, i}^{-s_{2}}, K_{4, i}\right) \cdot \mathbf{e}\left(H_{1, i}^{-s_{2}}, K_{5, i}\right) \cdot \mathbf{e}\left(H_{1, i}^{-s_{2}}, K_{6, i}\right)= \\
& =\Lambda^{s_{2}} \cdot \mathbf{e}\left(\mathrm{ct}_{2}, K_{B}^{-1}\right) \cdot \mathbf{e}(h, g)^{\left(\lambda_{1} s_{3}+\lambda_{2} s_{4}\right)\langle\boldsymbol{x}, \boldsymbol{v}\rangle}
\end{aligned}
$$

Plugging this into the decryption algorithm we get

$$
\Upsilon=m \cdot \mathbf{e}(h, g)^{\left(\lambda_{1} s_{3}+\lambda_{2} s_{4}\right)\langle\boldsymbol{x}, \boldsymbol{v}\rangle}, \Upsilon^{\prime}=m \cdot \mathbf{e}(h, g)^{\left(\lambda_{1} s_{3}^{\prime}+s_{4} \lambda_{2}\right)\langle\boldsymbol{x}, \boldsymbol{v}\rangle}
$$

First note that it cannot happen that $\mathrm{ct}_{7} \neq \mathrm{ct}_{7}^{\prime}$ for honestly generated ciphertexts. Clearly, $\langle\boldsymbol{x}, \boldsymbol{v}\rangle=0 \Rightarrow\left(\Upsilon=\Upsilon^{\prime}=m\right)$. All we need to check is thus that if $\langle\boldsymbol{x}, \boldsymbol{v}\rangle \neq 0$, we get output $\perp$. We could only get a wrong output if it happens that $\Upsilon=\Upsilon^{\prime}$, but this is impossible since it implies (using $\lambda_{1} \neq 0, s_{3} \neq s_{3}^{\prime}$ )

$$
\mathbf{e}(h, g)^{\left(\lambda_{1} s_{3}-\lambda_{1} s_{3}^{\prime}\right)\langle\boldsymbol{x}, \boldsymbol{v}\rangle}=1_{\mathbb{G}_{T}} \Rightarrow \lambda_{1}\left(s_{3}-s_{3}^{\prime}\right)\langle\boldsymbol{x}, \boldsymbol{v}\rangle \equiv_{p} 0 \Rightarrow\langle\boldsymbol{x}, \boldsymbol{v}\rangle \equiv_{p} 0 .
$$

\subsection{Security Reduction to DLin and DBDH}

In this section we prove our IPE scheme is IND-Secure under the standard computational assumptions.

Theorem 1. The IPE scheme IP of Construction 1 is IND-Secure if the DBDH and DLin assumptions hold relative to GroupGen.

To prove the theorem we define a series of hybrid experiments $\mathrm{H}_{0}, \ldots, \mathrm{H}_{12}$ in which $\mathrm{H}_{0}$ corresponds to the real experiment with challenge bit $b=0$ and $\mathrm{H}_{12}$ corresponds to the real experiment with challenge bit $b=1$, and we show that they are computationally indistinguishable. We provide the full proof of Theorem 1 in the full version of this paper [25].

- Hybrid $\mathrm{H}_{0}$ : this hybrid is identical to the real game with challenge bit $b=0$.

Precisely, the ciphertext is computed for message $m_{0}$ and vector $\boldsymbol{x}$ as follows:

$$
\begin{aligned}
\mathrm{ct}= & \left(g^{s_{2}}, h^{s_{1}},\left\{W_{b, i}^{s_{1}} \cdot F_{b, i}^{s_{2}} \cdot U_{b}^{x_{i} s_{3}}, T_{b, i}^{s_{1}} \cdot H_{b, i}^{s_{2}} \cdot V_{b}^{x_{i} s_{4}}\right\}_{b \in[2], i \in[n]}, \mathbf{e}\left(g^{s_{3}}, g^{s_{4}}\right),\right. \\
& \left.\Lambda^{-s_{2}} \cdot m_{0}\right) \\
\mathrm{ct}^{\prime}= & \left(g^{s_{2}^{\prime}}, h^{s_{1}^{\prime}},\left\{W_{b, i}^{s_{1}^{\prime}} \cdot F_{b, i}^{s_{2}^{\prime}} \cdot U_{b}^{x_{i} s_{3}^{\prime}}, T_{b, i}^{s_{1}^{\prime}} \cdot H_{b, i}^{s_{2}^{\prime}} \cdot V_{b}^{x_{i} s_{4}}\right\}_{b \in[2], i \in[n]}, \mathbf{e}\left(g^{s_{3}^{\prime}}, g^{s_{4}}\right),\right. \\
& \left.\Lambda^{-s_{2}^{\prime}} \cdot m_{0}\right)
\end{aligned}
$$


- Hybrid $\mathrm{H}_{1}$ : this hybrid is identical to the previous hybrid except that instead of $\mathbf{e}(g, g)^{s_{3} s_{4}}, \mathbf{e}(g, g)^{s_{3}^{\prime} s_{4}}$, the ciphertext contains two random elements $R_{1}, R_{1}^{\prime} \leftarrow \mathbb{G}_{T}$. Precisely, the ciphertext is computed as follows:

$$
\begin{aligned}
\mathrm{ct}= & \left(g^{s_{2}}, h^{s_{1}}\left\{W_{b, i}^{s_{1}} \cdot F_{b, i}^{s_{2}} \cdot U_{b}^{x_{i} s_{3}}, T_{b, i}^{s_{1}} \cdot H_{b, i}^{s_{2}} \cdot V_{b}^{x_{i} s_{4}}\right\}_{b \in[2], i \in[n]}, R_{1},\right. \\
& \left.\Lambda^{-s_{2}} \cdot m_{0}\right) \\
\mathrm{ct}^{\prime}= & \left(g^{s_{2}^{\prime}}, h^{s_{1}^{\prime}},\left\{W_{b, i}^{s_{1}^{\prime}} \cdot F_{b, i}^{s_{2}^{\prime}} \cdot U_{b}^{x_{i} s_{3}^{\prime}}, T_{b, i}^{s_{1}^{\prime}} \cdot H_{b, i}^{s_{2}^{\prime}} \cdot V_{b}^{x_{i} s_{4}}\right\}_{b \in[2], i \in[n]}, R_{1}^{\prime},\right. \\
& \left.\Lambda^{-s_{2}^{\prime}} \cdot m_{0}\right)
\end{aligned}
$$

- Hybrid $\mathrm{H}_{2}$ : this hybrid is identical to the previous hybrid except that instead of $\Lambda^{-s_{2}} \cdot m_{0}, \Lambda^{-s_{2}^{\prime}} \cdot m_{0}$, the ciphertext contains two random elements $R, R^{\prime} \leftarrow$ $\mathbb{G}_{T}$. Precisely, the ciphertext is computed as follows:

$$
\begin{aligned}
& \mathrm{ct}=\left(g^{s_{2}}, h^{s_{1}},\left\{W_{b, i}^{s_{1}} \cdot F_{b, i}^{s_{2}} \cdot U_{b}^{x_{i} s_{3}}, T_{b, i}^{s_{1}} \cdot H_{b, i}^{s_{2}} \cdot V_{b}^{x_{i} s_{4}}\right\}_{b \in[2], i \in[n]}, R_{1}, R\right) \\
& \mathrm{ct}^{\prime}=\left(g^{s_{2}^{\prime}}, h^{s_{1}^{\prime}},\left\{W_{b, i}^{s_{1}^{\prime}} \cdot F_{b, i}^{s_{2}^{\prime}} \cdot U_{b}^{x_{i} s_{3}^{\prime}}, T_{b, i}^{s_{1}^{\prime}} \cdot H_{b, i}^{s_{2}^{\prime}} \cdot V_{b}^{x_{i} s_{4}}\right\}_{b \in[2], i \in[n]}, R_{1}^{\prime}, R^{\prime}\right)
\end{aligned}
$$

- Hybrid $\mathrm{H}_{3}$ : this hybrid is identical to the previous hybrid except that instead of $T_{b, i}^{s_{1}} \cdot H_{b, i}^{s_{2}} \cdot V_{b}^{x_{i} s_{4}}, T_{b, i}^{s_{1}^{\prime}} \cdot H_{b, i}^{s_{2}^{\prime}} \cdot V_{b}^{x_{i} s_{4}}$, the ciphertext contains $T_{b, i}^{s_{1}} \cdot H_{b, i}^{s_{2}}, T_{b, i}^{s_{1}^{\prime}} \cdot H_{b, i}^{s_{2}^{\prime}}$. Precisely, the ciphertext is computed as follows:

$$
\begin{aligned}
& \mathrm{ct}=\left(g^{s_{2}}, h^{s_{1}},\left\{W_{b, i}^{s_{1}} \cdot F_{b, i}^{s_{2}} \cdot U_{b}^{x_{i} s_{3}}, T_{b, i}^{s_{1}} \cdot H_{b, i}^{s_{2}}\right\}_{b \in[2], i \in[n]}, R_{1}, R\right) \\
& \mathrm{ct}^{\prime}=\left(g^{s_{2}^{\prime}}, h^{s_{1}^{\prime}},\left\{W_{b, i}^{s_{1}^{\prime}} \cdot F_{b, i}^{s_{2}^{\prime}} \cdot U_{b}^{x_{i} s_{3}^{\prime}}, T_{b, i}^{s_{1}^{\prime}} \cdot H_{b, i}^{s_{2}^{\prime}}\right\}_{b \in[2], i \in[n]}, R_{1}^{\prime}, R^{\prime}\right)
\end{aligned}
$$

- Hybrid $\mathrm{H}_{4}$ : this hybrid is identical to the previous hybrid except that instead of $T_{b, i}^{s_{1}} \cdot H_{b, i}^{s_{2}}, T_{b, i}^{s_{1}^{\prime}} \cdot H_{b, i}^{s_{2}^{\prime}}$, the ciphertext contains $T_{b, i}^{s_{1}} \cdot H_{b, i}^{s_{2}} \cdot V_{b}^{y_{i} s_{4}}, T_{b, i}^{s_{1}^{\prime}} \cdot H_{b, i}^{s_{2}^{\prime}} \cdot V_{b}^{y_{i} s_{4}}$. Precisely, the ciphertext is computed as follows:

$$
\begin{aligned}
& \mathrm{ct}=\left(g^{s_{2}}, h^{s_{1}},\left\{W_{b, i}^{s_{1}} \cdot F_{b, i}^{s_{2}} \cdot U_{b}^{x_{i} s_{3}}, T_{b, i}^{s_{1}} \cdot H_{b, i}^{s_{2}} \cdot V_{b}^{y_{i} s_{4}}\right\}_{b \in[2], i \in[n]}, R_{1}, R\right) \\
& \mathrm{ct}^{\prime}=\left(g^{s_{2}^{\prime}}, h^{s_{1}^{\prime}},\left\{W_{b, i}^{s_{1}^{\prime}} \cdot F_{b, i}^{s_{2}^{\prime}} \cdot U_{b}^{x_{i} s_{3}^{\prime}}, T_{b, i}^{s_{1}^{\prime}} \cdot H_{b, i}^{s_{2}^{\prime}} \cdot V_{b}^{y_{i} s_{4}}\right\}_{b \in[2], i \in[n]}, R_{1}^{\prime}, R^{\prime}\right)
\end{aligned}
$$

- Hybrid $\mathrm{H}_{5}: \mathrm{CT}_{6}=\left(\mathrm{ct}, \mathrm{ct}^{\prime}\right)$, This hybrid is identical to the previous hybrid except that the power of $V_{b}$ in ct is $s_{4}$ and its power in $\mathrm{ct}^{\prime}$ is $s_{4}^{\prime}$. Precisely, the ciphertext is computed as follows:

$$
\begin{aligned}
& \mathrm{ct}=\left(g^{s_{2}}, h^{s_{1}},\left\{W_{b, i}^{s_{1}} \cdot F_{b, i}^{s_{2}} \cdot U_{b}^{x_{i} s_{3}}, T_{b, i}^{s_{1}} \cdot H_{b, i}^{s_{2}} \cdot V_{b}^{y_{i} s_{4}}\right\}_{b \in[2], i \in[n]}, R_{1}, R\right) \\
& \mathrm{ct}^{\prime}=\left(g^{s_{2}^{\prime}}, h^{s_{1}^{\prime}},\left\{W_{b, i}^{s_{1}^{\prime}} \cdot F_{b, i}^{s_{2}^{\prime}} \cdot U_{b}^{x_{i} s_{3}^{\prime}}, T_{b, i}^{s_{1}^{\prime}} \cdot H_{b, i}^{s_{2}^{\prime}} \cdot V_{b}^{y_{i} s_{4}^{\prime}}\right\}_{b \in[2], i \in[n]}, R_{1}^{\prime}, R^{\prime}\right)
\end{aligned}
$$

- Hybrid $\mathrm{H}_{6}$ : this hybrid is identical to the previous hybrid except that $s_{3}=s_{3}^{\prime}$. Precisely:

$$
\begin{aligned}
& \mathrm{ct}=\left(g^{s_{2}}, h^{s_{1}},\left\{W_{b, i}^{s_{1}} \cdot F_{b, i}^{s_{2}} \cdot U_{b}^{x_{i} s_{3}}, T_{b, i}^{s_{1}} \cdot H_{b, i}^{s_{2}} \cdot V_{b}^{y_{i} s_{4}}\right\}_{b \in[2], i \in[n]}, R_{1}, R\right) \\
& \mathrm{ct}^{\prime}=\left(g^{s_{2}^{\prime}}, h^{s_{1}^{\prime}},\left\{W_{b, i}^{s_{1}^{\prime}} \cdot F_{b, i}^{s_{2}^{\prime}} \cdot U_{b}^{x_{i} s_{3}}, T_{b, i}^{s_{1}^{\prime}} \cdot H_{b, i}^{s_{2}^{\prime}} \cdot V_{b}^{y_{i} s_{4}^{\prime}}\right\}_{b \in[2], i \in[n]}, R_{1}^{\prime}, R^{\prime}\right)
\end{aligned}
$$


- Hybrid $\mathrm{H}_{7}$ : This hybrid is identical to the previous hybrid except we replace $s_{3}$ with 0 .

$$
\begin{aligned}
& \mathrm{ct}=\left(g^{s_{2}}, h^{s_{1}},\left\{W_{b, i}^{s_{1}} \cdot F_{b, i}^{s_{2}}, T_{b, i}^{s_{1}} \cdot H_{b, i}^{s_{2}} \cdot V_{b}^{y_{i} s_{4}}\right\}_{b \in[2], i \in[n]}, R_{1}, R\right) \\
& \mathrm{ct}^{\prime}=\left(g^{s_{2}^{\prime}}, h^{s_{1}^{\prime}},\left\{W_{b, i}^{s_{1}^{\prime}} \cdot F_{b, i}^{s_{2}^{\prime}}, T_{b, i}^{s_{1}^{\prime}} \cdot H_{b, i}^{s_{2}^{\prime}} \cdot V_{b}^{y_{i} s_{4}^{\prime}}\right\}_{b \in[2], i \in[n]}, R_{1}^{\prime}, R^{\prime}\right)
\end{aligned}
$$

- Hybrid $\mathrm{H}_{8}$ : This hybrid is identical to the previous hybrid except that instead of $W_{b, i}^{s_{1}} \cdot F_{b, i}^{s_{2}}, W_{b, i}^{s_{1}^{\prime}} \cdot F_{b, i}^{s_{2}^{\prime}}$, we set $W_{b, i}^{s_{1}} \cdot F_{b, i}^{s_{2}} \cdot U_{b}^{y_{i} s_{3}}, W_{b, i}^{s_{1}^{\prime}} \cdot F_{b, i}^{s_{2}^{\prime}} \cdot U_{b}^{y_{i} s_{3}}$. Precisely:

$$
\begin{aligned}
& \mathrm{ct}=\left(g^{s_{2}}, h^{s_{1}},\left\{W_{b, i}^{s_{1}} \cdot F_{b, i}^{s_{2}} \cdot U_{b}^{y_{i} s_{3}}, T_{b, i}^{s_{1}} \cdot H_{b, i}^{s_{2}} \cdot V_{b}^{y_{i} s_{4}}\right\}_{b \in[2], i \in[n]}, R_{1}, R\right) \\
& \mathrm{ct}^{\prime}=\left(g^{s_{2}^{\prime}}, h^{s_{1}^{\prime}},\left\{W_{b, i}^{s_{1}^{\prime}} \cdot F_{b, i}^{s_{2}^{\prime}} \cdot U_{b}^{y_{i} s_{3}}, T_{b, i}^{s_{1}^{\prime}} \cdot H_{b, i}^{s_{2}^{\prime}} \cdot V_{b}^{y_{i} s_{4}^{\prime}}\right\}_{b \in[2], i \in[n]}, R_{1}^{\prime}, R^{\prime}\right)
\end{aligned}
$$

- Hybrid $\mathrm{H}_{9}$ : this hybrid is identical to the previous hybrid except that instead of $W_{b, i}^{s_{1}} \cdot F_{b, i}^{s_{2}}, W_{b, i}^{s_{1}^{\prime}} \cdot F_{b, i}^{s_{2}^{\prime}}$, we set $W_{b, i}^{s_{1}} \cdot F_{b, i}^{s_{2}} \cdot U_{b}^{y_{i} s_{3}}, W_{b, i}^{s_{1}^{\prime}} \cdot F_{b, i}^{s_{2}^{\prime}} \cdot U_{b}^{y_{i} s_{3}^{\prime}}$. Precisely:

$$
\begin{aligned}
& \mathrm{ct}=\left(g^{s_{2}}, h^{s_{1}},\left\{W_{b, i}^{s_{1}} \cdot F_{b, i}^{s_{2}} \cdot U_{b}^{y_{i} s_{3}}, T_{b, i}^{s_{1}} \cdot H_{b, i}^{s_{2}} \cdot V_{b}^{y_{i} s_{4}}\right\}_{b \in[2], i \in[n]}, R_{1}, R\right) \\
& \mathrm{ct}^{\prime}=\left(g^{s_{2}^{\prime}}, h^{s_{1}^{\prime}},\left\{W_{b, i}^{s_{1}^{\prime}} \cdot F_{b, i}^{s_{2}^{\prime}} \cdot U_{b}^{y_{i} s_{3}^{\prime}}, T_{b, i}^{s_{1}^{\prime}} \cdot H_{b, i}^{s_{2}^{\prime}} \cdot V_{b}^{y_{i} s_{4}^{\prime}}\right\}_{b \in[2], i \in[n]}, R_{1}^{\prime}, R^{\prime}\right)
\end{aligned}
$$

- Hybrid $\mathrm{H}_{10}$ : this hybrid is identical to the previous hybrid except that instead of $W_{b, i}^{s_{1}} \cdot F_{b, i}^{s_{2}}, W_{b, i}^{s_{1}^{\prime}} \cdot F_{b, i}^{s_{2}^{\prime}}$, we set $W_{b, i}^{s_{1}} \cdot F_{b, i}^{s_{2}} \cdot U_{b}^{y_{i} s_{3}}, W_{b, i}^{s_{1}^{\prime}} \cdot F_{b, i}^{s_{2}^{\prime}} \cdot U_{b}^{y_{i} s_{3}^{\prime}}$. Precisely:

$$
\begin{aligned}
& \mathrm{ct}=\left(g^{s_{2}}, h^{s_{1}},\left\{W_{b, i}^{s_{1}} \cdot F_{b, i}^{s_{2}} \cdot U_{b}^{y_{i} s_{3}}, T_{b, i}^{s_{1}} \cdot H_{b, i}^{s_{2}} \cdot V_{b}^{y_{i} s_{4}}\right\}_{b \in[2], i \in[n]}, R_{1}, R\right) \\
& \mathrm{ct}^{\prime}=\left(g^{s_{2}^{\prime}}, h^{s_{1}^{\prime}},\left\{W_{b, i}^{s_{1}^{\prime}} \cdot F_{b, i}^{s_{2}^{\prime}} \cdot U_{b}^{y_{i} s_{3}^{\prime}}, T_{b, i}^{s_{1}^{\prime}} \cdot H_{b, i}^{s_{2}^{\prime}} \cdot V_{b}^{y_{i} s_{4}}\right\}_{b \in[2], i \in[n]}, R_{1}^{\prime}, R^{\prime}\right)
\end{aligned}
$$

- Hybrid $\mathrm{H}_{11}$ : this hybrid is identical to the previous hybrid except that instead of choosing $R, R^{\prime} \leftarrow \mathbb{G}_{T}$, we set $R=\Lambda^{-s_{2}} \cdot m_{1}, R^{\prime}=\Lambda^{-s_{2}^{\prime}} \cdot m_{1}$. Precisely, the ciphertext is computed as follows:

$$
\begin{aligned}
& \mathrm{ct}=\left(g^{s_{2}}, h^{s_{1}},\left\{W_{b, i}^{s_{1}} \cdot F_{b, i}^{s_{2}} \cdot U_{b}^{y_{i} s_{3}}, T_{b, i}^{s_{1}} \cdot H_{b, i}^{s_{2}} \cdot V_{b}^{y_{i} s_{4}}\right\}_{b \in[2], i \in[n]}, R_{1}, \Lambda^{-s_{2}} \cdot m_{1}\right) \\
& \mathrm{ct}^{\prime}=\left(g^{s_{2}^{\prime}}, h^{s_{1}^{\prime}},\left\{W_{b, i}^{s_{1}^{\prime}} \cdot F_{b, i}^{s_{2}^{\prime}} \cdot U_{b}^{y_{i} s_{3}^{\prime}}, T_{b, i}^{s_{1}^{\prime}} \cdot H_{b, i}^{s_{2}^{\prime}} \cdot V_{b}^{y_{i} s_{4}}\right\}_{b \in[2], i \in[n]}, R_{1}^{\prime}, \Lambda^{-s_{2}^{\prime}} \cdot m_{1}\right)
\end{aligned}
$$

- Hybrid $\mathrm{H}_{12}$ : this hybrid is identical to the previous hybrid except that instead of $R_{1}, R_{1}^{\prime}$, we set $\mathbf{e}\left(g^{s_{3}}, g^{s_{4}}\right), \mathbf{e}\left(g^{s_{3}^{\prime}}, g^{s_{4}}\right)$, which is identical to the real game with challenge bit $b=1$, in particular for message $m_{1}$ and vector $\boldsymbol{y}$. Precisely, the ciphertext is computed as follows:

$$
\begin{aligned}
\mathrm{ct}= & \left(g^{s_{2}}, h^{s_{1}},\left\{W_{b, i}^{s_{1}} \cdot F_{b, i}^{s_{2}} \cdot U_{b}^{y_{i} s_{3}}, T_{b, i}^{s_{1}} \cdot H_{b, i}^{s_{2}} \cdot V_{b}^{y_{i} s_{4}}\right\}_{b \in[2], i \in[n]}, \mathbf{e}\left(g^{s_{3}}, g^{s_{4}}\right)\right. \\
& \left.\Lambda^{-s_{2}} \cdot m_{1}\right) \\
\mathrm{ct}^{\prime}= & \left(g^{s_{2}^{\prime}}, h^{s_{1}^{\prime}},\left\{W_{b, i}^{s_{1}^{\prime}} \cdot F_{b, i}^{s_{2}^{\prime}} \cdot U_{b}^{y_{i} s_{3}^{\prime}}, T_{b, i}^{s_{1}^{\prime}} \cdot H_{b, i}^{s_{2}^{\prime}} \cdot V_{b}^{y_{i} s_{4}}\right\}_{b \in[2], i \in[n]}, \mathbf{e}\left(g^{s_{3}^{\prime}}, g^{s_{4}}\right)\right. \\
& \left.\Lambda^{-s_{2}^{\prime}} \cdot m_{1}\right)
\end{aligned}
$$


Proposition 2. If the DLin assumption holds relative to GroupGen, then $\mathrm{H}_{0}$ is computationally indistinguishable from $\mathrm{H}_{1}$.

Proof. Let us assume there exists a PPT adversary $\mathcal{A}$ which distinguishes between $\mathrm{H}_{0}$ and $\mathrm{H}_{1}$ with non-negligible advantage. We describe a simulator $\mathcal{B}$ which uses $\mathcal{A}$, on input $\left(g, A=g^{\alpha}, B=g^{\beta}, C=g^{\tau}, D=g^{\alpha \eta}, Z\right) \in \mathbb{G}^{6}$, output 1 if $Z=g^{\beta(\eta+\tau)}$ and 0 if $Z$ is a random element in $\mathbb{G}$. $\mathcal{B}$ interacts with $\mathcal{A}$ as follows:

SetUp Phase. The adversary $\mathcal{A}$ sends to the simulator, $\mathcal{B}$, two vectors $\boldsymbol{x}, \boldsymbol{y} \in$ $\mathbb{Z}_{p}^{n}$. The simulator picks $g^{\prime} \leftarrow \mathbb{G}$ and $\tilde{\Omega}, k, \tilde{\delta}_{b}, \theta_{b},\left\{w_{1, i}, \tilde{t}_{1, i}, f_{b, i}, h_{b, i}\right\}_{i \in[n], b \in[2]} \leftarrow$ $\mathbb{Z}_{p}$, compute $\left\{w_{2, i}, \tilde{t}_{2, i}\right\}_{i \in[n]}$ such that for each $i, \tilde{\Omega}=\tilde{\delta}_{1} w_{2, i}-\tilde{\delta}_{2} w_{1, i}=\theta_{1} \tilde{t}_{2, i}-$ $\theta_{2} \tilde{t}_{1, i}$. Compute the master public key components as follows and returns it:

$$
\begin{aligned}
& \left\{W_{b, i}=g^{w_{b, i}}, F_{b, i}=g^{f_{b, i}}\right\}_{b \in[2], i \in[n]},\left\{U_{b}=A^{\tilde{\delta}_{b}}\right\}_{b \in[2]}, h=A^{\tilde{\Omega}}, \Lambda=\mathbf{e}\left(g, g^{\prime}\right) . \\
& \left\{T_{b, i}=A^{\tilde{t}_{b, i}}, H_{b, i}=g^{h_{b, i}}\right\}_{b \in[2], i \in[n]},\left\{V_{b}=g^{\theta_{b}}\right\}_{b \in[2]}, K_{1}=g^{k}, K_{2}=g^{\prime \frac{1}{k}}
\end{aligned}
$$

By doing so, $\mathcal{B}$ implicitly sets $\delta_{b}=\alpha \tilde{\delta}_{b}, t_{b, i}=\alpha \tilde{t}_{b, i}$ for $b \in[2], i \in[n]$ and $\Omega=\alpha \tilde{\Omega}$, which shows that each element of the master public key is independently and uniformly distributed in $\mathbb{Z}_{p}$. Also notice that for each $i \in[n]$, we have: $\delta_{1} w_{2, i}-$ $\delta_{2} w_{1, i}=\alpha \tilde{\delta}_{1} w_{2, i}-\alpha \tilde{\delta}_{2} w_{1, i}=\theta_{1} \alpha \tilde{t}_{2, i}-\theta_{2} \alpha \tilde{t}_{1, i}=\theta_{1} t_{2, i}-\theta_{2} t_{1, i}=\alpha \tilde{\Omega}=\Omega$. hence the output has the same structure as the output of the real setup algorithm.

Token Query Phase. All the secret parameters except $\left\{\delta_{b}, t_{b, i}\right\}_{b \in[2], i \in[n]}, \Omega$ are known by $\mathcal{B}$. When $\mathcal{A}$ asks for a query for a vector $\boldsymbol{v}, \mathcal{B}$ picks $\lambda_{1}, \tilde{\lambda}_{2},\left\{\tilde{r}_{i}, \Phi_{i}\right\}_{i \in[n]} \leftarrow \mathbb{Z}_{p}^{\star}$. In generating Tok $_{\boldsymbol{v}}$, the simulator implicitly sets $\lambda_{2}=\alpha \tilde{\lambda}_{2}, r_{i}=\alpha \tilde{r}_{i}$ which are independently and uniformly distributed in $\mathbb{Z}_{p}^{\star}$. Token elements are set as follows:

$$
\begin{aligned}
& K_{3, i}=A^{-\tilde{\delta}_{2} r_{i}} \cdot g^{\lambda_{1} v_{i} w_{2, i} x_{i}}=(\text { by the above settings })=g^{-\delta_{2} r_{i}} \cdot g^{v_{i} w_{2, i} \lambda_{1}} . \\
& K_{5, i}=g^{-\theta_{2} \phi_{i}} \cdot A^{\lambda_{2} v_{i} \tilde{t}_{2, i} x_{i}}=(\text { by the above settings })=g^{-\theta_{2} \phi_{i}} \cdot g^{\lambda_{2} v_{i} t_{2, i} x_{i}} . \\
& \text { Similarly, } K_{4, i}=A^{\tilde{\delta}_{1} r_{i}} \cdot g^{-\lambda_{1} v_{i} w_{1, i} x_{i}}, K_{6, i}=g^{\theta_{1} r_{i}} \cdot A^{-\lambda_{2} v_{i} \tilde{t}_{1, i} x_{i}} \cdot \\
& K_{B}=\prod_{i=1}^{n} A^{-r_{i}} g^{-\Phi_{i}}=\prod_{i=1}^{n} g^{-\left(\alpha \tilde{r}_{i}+\Phi_{i}\right)}=\prod_{i=1}^{n} g^{-\left(r_{i}+\Phi_{i}\right)} .
\end{aligned}
$$

$\mathcal{B}$ knows $\left\{f_{b, i}, h_{b, i}\right\}_{b \in[2], i \in[n]}$, hence it can compute $K_{A}$.

Generating the Challenge Ciphertext. $\mathcal{A}$ sends message $m_{0}$ to $\mathcal{B}$. To generate a challenge ciphertext, $\mathcal{B}$ picks $s_{1}, s_{2}, s_{1}^{\prime}, s_{2}^{\prime}, \tilde{s}_{3}, \tilde{s}_{4}, \tilde{s}_{3^{\prime}} \leftarrow \mathbb{Z}_{p}^{\star}$ such that $\tilde{s}_{3} \neq \tilde{s}_{3}^{\prime}$. $\mathcal{B}$ implicitly sets $s_{3}=\eta \tilde{s}_{3}, s_{4}=\beta \tilde{s}_{4}$ and computes the ciphertext as follows: 


$$
\begin{array}{ll}
\mathrm{ct}_{1}=g^{s_{2}}, \mathrm{ct}_{1}^{\prime}=g^{s_{2}^{\prime}} & , \mathrm{ct}_{2}=h^{s_{1}}, \mathrm{ct}_{2}^{\prime}=h^{s_{1}^{\prime}} \cdot \\
\mathrm{ct}_{3, i}=W_{1, i}^{s_{1}} \cdot F_{1, i}^{s_{2}} \cdot D^{\tilde{\delta}_{1} \tilde{s}_{3} x_{i}} & , \mathrm{ct}_{3, i}^{\prime}=W_{1, i}^{s_{1}^{\prime}} \cdot F_{1, i}^{s_{2}^{\prime}} \cdot D^{\tilde{\delta}_{1} x_{i} \tilde{s}_{3}^{\prime}} . \\
\mathrm{ct}_{4, i}=W_{2, i}^{s_{1}} \cdot F_{2, i}^{s_{2}} \cdot D^{\tilde{\delta}_{2} \tilde{s}_{3} x_{i}} & , \mathrm{ct}_{4, i}^{\prime}=W_{2, i}^{s_{1}^{\prime}} \cdot F_{2, i}^{s_{2}^{\prime}} \cdot D^{\tilde{\delta}_{2} x_{i} \tilde{s}_{3}^{\prime}} . \\
\mathrm{ct}_{5, i}=T_{1, i}^{s_{1}} \cdot H_{1, i}^{s_{2}} \cdot B^{\theta_{1} \tilde{s}_{4} x_{i}} & , \mathrm{ct}_{5, i}^{\prime}=T_{1, i}^{s_{1}^{\prime}} \cdot H_{1, i}^{s_{2}^{\prime}} \cdot B^{\theta_{1} \tilde{s}_{4} x_{i}} \\
\mathrm{ct}_{6, i}=T_{2, i}^{s_{1}} \cdot H_{2, i}^{s_{2}} \cdot B^{\theta_{2} \tilde{s}_{4} x_{i}} & , \mathrm{ct}_{6, i}^{\prime}=T_{2, i}^{s_{1}^{\prime}} \cdot H_{2, i}^{s_{2}^{\prime}} \cdot B^{\theta_{2} \tilde{s}_{4} x_{i}} \\
\mathrm{ct}_{7}=\left(\frac{\mathbf{e}(Z, g)}{\mathbf{e}(B, C)}\right)^{\tilde{s}_{3} \tilde{s}_{4}}, & , \mathrm{ct}_{7}^{\prime}=\left(\frac{\mathbf{e}(Z, g)}{\mathbf{e}(B, C)}\right)^{\tilde{s}_{3^{\prime}} \tilde{s}_{4}}, \\
\mathrm{ct}_{8}=\mathbf{e}\left(g, g^{\prime}\right)^{-s_{2}} \cdot m_{0} & , \mathrm{ct}_{8}^{\prime}=\mathbf{e}\left(g, g^{\prime}\right)^{-s_{2}^{\prime}} \cdot m_{0}
\end{array}
$$

Since $D^{\tilde{\delta}_{b} x_{i} \tilde{s}_{3}}=g^{\alpha \tilde{\delta}_{b} \eta \tilde{s}_{3} x_{i}}=U_{b}^{x_{i} s_{3}}, B^{\theta_{b} \tilde{s}_{4} x_{i}}=V_{1}^{\beta \tilde{s}_{4} x_{i}}=V_{b}^{s_{4} x_{i}}$, for each $i \in[n]$ the values $\mathrm{ct}_{3, i}, \mathrm{ct}_{3, i}^{\prime}, \ldots, \mathrm{ct}_{6, i}, \mathrm{ct}_{6, i}^{\prime}$ are computed properly.

Analysing the Game: Let us analyze the two events, $Z=g^{\beta(\tau+\eta)}$ or $Z \leftarrow \mathbb{G}$ :

$$
\begin{aligned}
& \text { - } Z=g^{\beta(\tau+\eta)} \Rightarrow \frac{\mathbf{e}(Z, g)}{\mathbf{e}(B, C)}=\frac{\mathbf{e}\left(g^{\beta(\tau+\eta)}, g\right)}{\mathbf{e}\left(g^{\beta}, g^{\tau}\right)}=\frac{\mathbf{e}\left(g^{\beta}, g^{\tau}\right) \cdot \mathbf{e}\left(g^{\beta}, g^{\eta}\right)}{\mathbf{e}\left(g^{\beta}, g^{\tau}\right)}=\mathbf{e}\left(g^{\eta}, g^{\beta}\right) \\
& \Rightarrow \mathrm{ct}_{7}=\left(\frac{\mathbf{e}(Z, g)}{\mathbf{e}(B, C)}\right)^{\tilde{s}_{3} \tilde{s}_{4}}=\mathbf{e}\left(g^{\eta \tilde{s}_{3}}, g^{\beta \tilde{s}_{4}}\right)=\mathbf{e}\left(g^{s_{3}}, g^{s_{4}}\right), \mathrm{ct}_{7}^{\prime}=\mathbf{e}\left(g^{s_{3}^{\prime}}, g^{s_{4}}\right) \\
& \Rightarrow \mathcal{A} \text { interacting with } \mathrm{H}_{0} .
\end{aligned}
$$

- $Z \leftarrow \mathbb{G} \Rightarrow \mathrm{ct}_{7}, \mathrm{ct}_{7}^{\prime}$ random elements in $\mathbb{G}_{T} \Rightarrow \mathcal{A}$ interacting with $\mathrm{H}_{1}$.

Proposition 3. If the DBDH assumption holds relative to GroupGen, then $\mathrm{H}_{1}$ is computationally indistinguishable from $\mathrm{H}_{2}$.

Proposition 4. If the DLin assumption holds relative to GroupGen, then $\mathrm{H}_{2}$ is computationally indistinguishable from $\mathrm{H}_{3}$.

Proposition 5. If the DLin assumption holds relative to GroupGen, then $\mathrm{H}_{3}$ is computationally indistinguishable from $\mathrm{H}_{4}$.

The Propositions 3, 4, 5 are proved in the full version [25].

Proposition 6. If the DLin assumption holds relative to GroupGen, then $\mathrm{H}_{4}$ is computationally indistinguishable from $\mathrm{H}_{5}$.

Proof. The simulator takes as input $\left(g, A=g^{\alpha}, B=g^{\beta}, C=g^{\tau}, D=g^{\alpha \eta}, Z \stackrel{?}{=}\right.$ $\left.g^{\beta(\eta+\tau)}\right)$ and by interacting with the adversary $\mathcal{A}$, distinguish between the two cases $Z=g^{\beta(\eta+\tau)}$ and $Z \stackrel{\$}{\leftarrow} \mathbb{G}$, a random element of the group.

SetUp and Token Query Phase. $\mathcal{B}$ runs as in the SetUp phase and token query phase in Proposition 5. 
Generating the Challenge Ciphertext. $\mathcal{B}$ chooses random elements $\tilde{s}_{1}, \tilde{s}_{2}, \tilde{s}_{3}$, $\tilde{s}_{4}, \tilde{s}_{1}^{\prime}, \tilde{s}_{2}^{\prime}, \tilde{s}_{3}^{\prime}, k \leftarrow \mathbb{Z}_{p}^{\star}$ and computes the challenge ciphertext as follows:

$\bullet \mathrm{ct}_{1}=C \cdot g^{\tilde{s}_{2}}=g^{\tau+\tilde{s}_{2}} \Rightarrow s_{2}=\tau+\tilde{s}_{2}, \bullet \mathrm{ct}_{1}^{\prime}=C^{k} \cdot g^{\tilde{s}_{2 \prime}}=g^{k \tau+\tilde{s}_{2}^{\prime}} \Rightarrow s_{2}^{\prime}=k \tau+\tilde{s}_{2}$

- $\mathrm{ct}_{2}=D^{\tilde{\Omega}} \cdot A^{\tilde{\Omega} \tilde{s}_{1}}=\left(g^{\alpha \tilde{\Omega}}\right)^{\left(\eta+\tilde{s}_{1}\right)}=h^{\eta+\tilde{s}_{1}} \Rightarrow s_{1}=\eta+\tilde{s}_{1}$

$\bullet \mathrm{ct}_{2}^{\prime}=D^{k \tilde{\Omega}} \cdot A^{\tilde{\Omega} \tilde{s}_{1}^{\prime}}=\left(g^{\alpha \tilde{\Omega}}\right)^{\left(k \eta+\tilde{s}_{1}^{\prime}\right)}=h^{k \eta+\tilde{s}_{1}^{\prime}} \Rightarrow s_{1}^{\prime}=k \eta+\tilde{s}_{1}^{\prime}$

- $\mathrm{ct}_{3, i}=W_{1, i}^{\tilde{s}_{1}} \cdot F_{1, i}^{\tilde{s}_{2}} \cdot U_{1}^{\tilde{s}_{3} x_{i}} \cdot D^{\tilde{w}_{1, i}} \cdot C^{f_{1, i}}=W_{1, i}^{\tilde{s}_{1}} \cdot F_{1, i}^{\tilde{s}_{2}+\tau} \cdot U_{1}^{\tilde{s}_{3} x_{i}} \cdot g^{\eta \alpha \tilde{w}_{1, i}} \cdot F_{1, i}^{\tau}=$ $=W_{1, i}^{\tilde{s}_{1}} \cdot F_{1, i}^{\tilde{s}_{2}+\tau} \cdot U_{1}^{\tilde{s}_{3} x_{i}} \cdot g^{\eta\left(w_{1, i}-\beta \delta_{1} x_{i}\right)}=W_{1, i}^{\tilde{s}_{1}+\eta} \cdot F_{1, i}^{\tilde{s}_{2}+\tau} \cdot U_{1}^{\left(\tilde{s}_{3}-\eta \beta\right) x_{i}}$

$\Rightarrow s_{3}=-\eta \beta+\tilde{s}_{3}$

- $\mathrm{ct}_{4, i}=W_{2, i}^{\tilde{s}_{1}} \cdot F_{2, i}^{\tilde{s}_{2}} \cdot U_{2}^{\tilde{s}_{3} x_{i}} \cdot D^{\tilde{w}_{2, i}} \cdot C^{f_{2, i}},\left(\right.$ similar computation as $\left.\mathrm{ct}_{3, i}\right)$

- $\mathrm{ct}_{3, i}^{\prime}=W_{1, i}^{\tilde{s_{1}^{\prime}}} \cdot F_{1, i}^{\tilde{s_{2}^{\prime}}} \cdot U_{1}^{\tilde{s}_{3}^{\prime} x_{i}} \cdot D^{k \tilde{w}_{1, i}} \cdot C^{k f_{1, i}}=W_{1, i}^{\tilde{s}_{1}^{\prime}} \cdot F_{1, i}^{\tilde{s}_{2}^{\prime}} \cdot U_{1}^{\tilde{s}_{3}^{\prime} x_{i}} \cdot g^{k \eta \alpha \tilde{w}_{1, i}} \cdot F_{1, i}^{k \tau}$

$=W_{1, i}^{\tilde{s}_{1}^{\prime}} \cdot F_{1, i}^{\tilde{s}_{2}^{\prime}+k \tau} \cdot U_{1}^{\tilde{s}_{3}^{\prime} x_{i}} \cdot g^{k \eta\left(w_{1, i}-\beta \delta_{1} x_{i}\right)}=W_{1, i}^{\tilde{s}_{1}^{\prime}+k \eta} \cdot F_{1, i}^{\tilde{s}_{2}^{\prime}+k \tau} \cdot U_{1}^{\left(\tilde{s}_{3}^{\prime}-k \eta \beta\right) x_{i}}$

$\Rightarrow s_{3}^{\prime}=-k \eta \beta+\tilde{s}_{3}^{\prime}$

- $\mathrm{ct}_{4, i}^{\prime}=W_{2, i}^{\tilde{s}_{1}^{\prime}} \cdot F_{2, i}^{\tilde{s}_{2}^{\prime}} \cdot U_{2}^{\tilde{s}_{3}^{\prime} x_{i}} \cdot D^{k \tilde{w}_{2, i}} \cdot C^{k f_{2, i}},\left(\right.$ similar computation as $\left.\mathrm{ct}_{3, i}^{\prime}\right)$

• $\mathrm{ct}_{5, i}=T_{1, i}^{\tilde{s}_{1}} \cdot D^{\tilde{t}_{1, i}} \cdot H_{1, i}^{\tilde{s}_{2}} \cdot C^{\tilde{h}_{1, i}} \cdot Z^{\theta_{1} y_{i}} \cdot g^{\tilde{s}_{4} \theta_{1} y_{i}}$

• $\mathrm{ct}_{5, i}^{\prime}=T_{1, i}^{\tilde{s}_{1}^{\prime}} \cdot D^{k \tilde{t}_{1, i}} \cdot H_{1, i}^{\tilde{s}_{2}^{\prime}} \cdot C^{k \tilde{h}_{1, i}} \cdot Z^{k \theta_{1} y_{i}} \cdot g^{\tilde{s}_{4} \theta_{1} y_{i}}$

- $\mathrm{ct}_{6, i}=T_{2, i}^{\tilde{s}_{1}} \cdot D^{\tilde{t}_{2, i}} \cdot H_{2, i}^{\tilde{s}_{2}} \cdot C^{\tilde{h}_{2, i}} \cdot Z^{\theta_{2} y_{i}} \cdot g^{\tilde{s}_{4} \theta_{2} y_{i}}$

$\bullet \mathrm{ct}_{6, i}^{\prime}=T_{2, i}^{\tilde{s}_{1}^{\prime}} \cdot D^{k \tilde{t}_{2, i}} \cdot H_{2, i}^{\tilde{s}_{2}^{\prime}} \cdot C^{k \tilde{h}_{2, i}} \cdot Z^{k \theta_{2} y_{i}} \cdot g^{\tilde{s}_{4} \theta_{2} y_{i}}$

Analysis of the Game: First, notice that:

$$
\begin{aligned}
D^{\tilde{t}_{1, i}} & =g^{\eta \alpha \tilde{t}_{1, i}}=g^{\eta\left(t_{1, i}-\beta \theta_{1} y_{i}\right)}=T_{1, i}^{\eta} \cdot g^{-\beta \eta \theta_{1} y_{i}}, D^{k \tilde{t}_{1, i}}=T_{1, i}^{k \eta} \cdot g^{-k \beta \eta \theta_{1} y_{i}} \\
C^{\tilde{h}_{1, i}} & =g^{\tau\left(h_{1, i}-\beta \theta_{1} y_{i}\right)}=H_{1, i}^{\tau} \cdot g^{-\beta \tau \theta_{1} y_{i}}, C^{k \tilde{h}_{1, i}}=H_{1, i}^{k \tau} \cdot g^{-k \beta \tau \theta_{1} y_{i}} \Rightarrow \\
\mathrm{ct}_{5, i} & =T_{1, i}^{\tilde{s}_{1}} \cdot D^{\tilde{t}_{1, i}} \cdot H_{1, i}^{\tilde{s}_{2}} \cdot C^{\tilde{h}_{1, i}} \cdot\left(Z \cdot g^{\tilde{s}_{4}}\right)^{\theta_{1} y_{i}} \\
& =T_{1, i}^{\eta+\tilde{s}_{1}} \cdot H_{1, i}^{\tau+\tilde{s}_{2}} \cdot\left(g^{-\beta(\tau+\eta)} \cdot Z \cdot g^{\tilde{s}_{4}}\right)^{\theta_{1} y_{i}} \\
& =T_{1, i}^{s_{1}} \cdot H_{1, i}^{s_{2}} \cdot\left(g^{(-\beta(\tau+\eta))} \cdot Z \cdot g^{\tilde{s}_{4}}\right)^{\theta_{1} y_{i}} \\
\mathrm{ct}_{5, i}^{\prime} & =T_{1, i}^{s_{1}^{\prime}} \cdot H_{1, i}^{s_{2}^{\prime}} \cdot\left(g^{(-k \beta(\tau+\eta))} \cdot Z^{k} \cdot g^{\tilde{s}_{4}}\right)^{\theta_{1} y_{i}}
\end{aligned}
$$

If $Z=g^{\beta(\eta+\tau)} \Rightarrow\left\{\begin{array}{l}g^{-\beta(\tau+\eta)} \cdot Z \cdot g^{\tilde{s}_{4}}=g^{\tilde{s}_{4}} \Rightarrow \mathrm{ct}_{5, i}=T_{1, i}^{s_{1}} \cdot H_{1, i}^{s_{2}} \cdot U_{1}^{s_{4} y_{i}} \\ g^{(-k \beta(\tau+\eta))} \cdot Z^{k} \cdot g^{\tilde{s}_{4}}=g^{\tilde{s}_{4}} \Rightarrow \mathrm{ct}_{5, i}=T_{1, i}^{s_{1}^{\prime}} \cdot H_{1, i}^{s_{2}^{\prime}} \cdot U_{1}^{s_{4} y_{i}}\end{array}\right.$

$\Rightarrow$ The adversary interacts with hybrid $\mathrm{H}_{4}$

If $Z=g^{r}$

$$
\Rightarrow\left\{\begin{array}{l}
g^{-\beta(\tau+\eta)} \cdot Z \cdot g^{\tilde{s}_{4}}=g^{r+\tilde{s}_{4}} \Rightarrow \mathrm{ct}_{5, i}=T_{1, i}^{s_{1}} \cdot H_{1, i}^{s_{2}} \cdot U_{1}^{s_{4} y_{i}} \\
g^{(-k \beta(\tau+\eta))} \cdot Z^{k} \cdot g^{\tilde{s}_{4}}=g^{k r+\tilde{s}_{4}} \Rightarrow \mathrm{ct}_{5, i}=T_{1, i}^{s_{1}^{\prime}} \cdot H_{1, i}^{s_{2}^{\prime}} \cdot U_{1}^{s_{4}^{\prime} y_{i}}
\end{array}\right.
$$

$\Rightarrow$ The adversary interacts with hybrid $\mathrm{H}_{5}$. 


\section{Verifiable Inner-Product Encryption}

Firstly, we present a formal definition of a VIPE scheme. Essentially, VIPE is similar to IPE except that it is endowed with extra verification algorithms VrfyCT, VrfyTok and VrfyMPK.

Definition 5. A verifiable inner product encryption scheme for a message space $\mathcal{M}$ and for a family $\Sigma=\left\{\Sigma_{n}\right\}_{n>0}$ of vectors over some field is a tuple of PPT algorithms (here called VIP) VIP $=\{$ VIP.SetUp, VIP.TokGen, VIP.Enc, VIP.Dec, VIP.VrfyMPK, VIP.VrfyCT, VIP.VrfyTok\} with the syntax and properties below:

- VIP.SetUp $\left(1^{\lambda}, n\right) \rightarrow($ MPK, MSK): as for IPE.

- VIP.TokGen(MPK, MSK, $v) \longrightarrow$ Tok $_{v}$ : as for IPE.

- VIP.Enc(MPK, $\vec{x}, m) \rightarrow$ CT: as for IPE.

- VIP.Dec(MPK, Tok $\left.{ }_{v}, \mathrm{CT}\right) \rightarrow m \in \mathcal{M} \cup\{\perp\}$ : as for IPE.

- VIP.VrfyMPK(MPK) $\rightarrow\{0,1\}$ : this is a deterministic algorithm that outputs 1 if MPK was correctly generated, or outputs 0 otherwise.

- VIP.VrfyCT(MPK, CT $) \rightarrow\{0,1\}$ : this is a deterministic algorithm that outputs 1 if $\mathrm{CT}$ was correctly generated using the master public key on input some $m$ in the message space $\mathcal{M}$ and a vector $\boldsymbol{x}$, or outputs 0 otherwise.

- VIP.VrfyTok(MPK, $\boldsymbol{v}$, Tok $\left._{\boldsymbol{v}}\right) \longrightarrow\{0,1\}$ : this is a deterministic algorithm that outputs 1 if Tok $_{v}$ was correctly generated using the master secret key on input vector $\boldsymbol{v}$, or outputs 0 otherwise.

- Perfect correctness: as for IPE.

- Verifiability: VIP is verifiable if for all $\mathrm{MPK} \in\{0,1\}^{*}$, all $\mathrm{CT} \in\{0,1\}^{*}$, there exists $n>0,(\boldsymbol{x}, m) \in \Sigma_{n} \times \mathcal{M}$ such that for all $\boldsymbol{v} \in \Sigma_{n}$ and Tok $_{\boldsymbol{v}} \in\{0,1\}^{*}$, the following holds:

$$
\left(\begin{array}{l}
\text { VIP.VrfyMPK }(M P K)=1 \wedge \\
\text { VIP.VrfyCT }(M P K, C T)=1 \wedge \\
\text { VIP.VrfyTok }\left(M P K, v, T_{0 k}\right)=1
\end{array}\right) \Rightarrow \operatorname{Pr}\left[\begin{array}{l}
\operatorname{VIP.Dec}\left(M P K, T_{0 k}, C T\right) \\
=f_{v}(\boldsymbol{x}, m)
\end{array}\right]=1
$$

Intuitively verifiability states that each ciphertext (possibly with a maliciously generated public key) should be associated with a unique message $(\boldsymbol{x}, m)$ and decryption for a function $f_{v}$ using any possibly maliciously generated token Tok should result in $f_{\boldsymbol{v}}(\boldsymbol{x}, m)$ for the unique message associated with the ciphertext [2].

\subsection{Our Construction}

Our VIPE is based on a perfectly correct IPE (cf. our IPE scheme of Construction 1), a perfectly binding commitment scheme such as the commitment scheme proposed in [13] and NIWI proofs for some specific relations that will be detailed below.

Let $n \in \mathbb{N}$ be the vector length and $\lambda$ the security parameter. Let IP be a perfectly correct IPE scheme, Com be a perfectly binding commitment scheme and NIWI ${ }^{\text {mpk }}=\left\langle\mathcal{P}^{\text {mpk }}, \mathcal{V}^{\text {mpk }}\right\rangle$, NIWI ${ }^{\text {enc }}=\left\langle\mathcal{P}^{\text {enc }}, \mathcal{V}^{\text {enc }}\right\rangle$ and NIWI ${ }^{\text {tok }}=\left\langle\mathcal{P}^{\text {tok }}, \mathcal{V}^{\text {tok }}\right\rangle$ be NIWI proofs systems for, resp., the relations $R^{\mathrm{mpk}}$, $\mathrm{R}^{\mathrm{enc}}$ and $\mathrm{R}^{\text {tok }}$, that are essentially instantiations of analogous relations in [2]. The construction of these NIWI systems is provided in Sect. 5 . 
- $\mathrm{R}_{3 \mathrm{P}}^{\mathrm{mpk}}(\overbrace{\mathrm{mpk}}^{x}, \overbrace{\left(\mathrm{msk}, \mathrm{r}^{\mathrm{mpk}}\right)}^{w})=$ TRUE $\Longleftrightarrow(\mathrm{mpk}, \mathrm{msk})=\mathrm{IP} \cdot \operatorname{SetUp}\left(1^{\lambda}, n ; \mathrm{r}^{\mathrm{mpk}}\right)$

- $\mathrm{R}_{\mathrm{IP}}^{\mathrm{tok}}(\overbrace{(\mathrm{mpk}, t, \boldsymbol{v})}^{x}, \overbrace{\left(\mathrm{msk}, \mathrm{r}^{\mathrm{mpk}}, \mathrm{r}^{\text {token }}\right)}^{w})=$ TRUE

$\Longleftrightarrow\left(\begin{array}{c}\left(\mathrm{mpk},\left(\mathrm{msk}, \mathrm{r}^{\mathrm{mpk}}\right)\right) \in \mathrm{R}_{\mathrm{IP}}^{\mathrm{mpk}} \wedge \\ t=\text { IP.TokGen }\left(\mathrm{MSK}, \boldsymbol{v} ; \mathrm{r}^{\mathrm{tok}}\right)\end{array}\right)$

- $\mathrm{R}_{3 \mathrm{IP}}^{k, \mathrm{ct}}(\overbrace{\left(\left(\mathrm{ct}_{1}, \mathrm{mpk}_{1}\right), \ldots,\left(\mathrm{ct}_{k}, \mathrm{mpk}_{k}\right)\right)}, \overbrace{\left(\boldsymbol{x}, m, \mathrm{r}_{1}^{\text {enc }}, \ldots, \mathrm{r}_{k}^{\mathrm{enc}}\right)}^{\mathrm{en}})=$ TRUE, $k \in[4]$ $\Longleftrightarrow \forall i \in[k] \mathrm{ct}_{i}=$ IP.Enc $\left(\mathrm{mpk}_{i}, \boldsymbol{x}, m ; \mathrm{r}_{i}^{\mathrm{enc}}\right)$

- $\mathrm{R}^{\text {enc }}(x, w)=\operatorname{TRUE} \Longleftrightarrow \mathrm{P}_{1}^{\text {enc }}(x, w) \vee \mathrm{P}_{2}^{\text {enc }}(x, w)$, with $\mathrm{P}_{1}^{\text {enc }}\left(\left(\left\{c_{i}\right\}_{i \in[4]},\left\{a_{i}\right\}_{i \in[4]}, z_{0}, z_{1}\right),\left(m, \boldsymbol{x},\left\{\mathrm{r}_{i}^{\text {enc }}\right\}_{i \in[4]}, i_{1}, i_{2}, \mathrm{r}_{0}^{\text {com }}, \mathrm{r}_{1}^{\text {com }}\right)\right)=$ TRUE $\Longleftrightarrow\left(\left(\left(c_{1}, a_{1}\right), \ldots,\left(c_{4}, a_{4}\right)\right),\left(\boldsymbol{x}, m,\left\{\mathrm{r}_{i}^{\text {enc }}\right\}_{i \in[4]}\right)\right) \in \mathrm{R}_{\mathrm{IP}}^{4, \mathrm{ct}}$ $\mathrm{P}_{2}^{\text {enc }}\left(\left(\left\{c_{i}\right\}_{i \in[4]},\left\{a_{i}\right\}_{i \in[4]}, z_{0}, z_{1}\right),\left(m, \boldsymbol{x},\left\{\mathrm{r}_{i}^{\text {enc }}\right\}_{i \in[4]}, i_{1}, i_{2}, \mathrm{r}_{0}^{\text {com }}, \mathrm{r}_{1}^{\text {com }}\right)\right)=$ TRUE $\Longleftrightarrow\left(\begin{array}{c}i_{1}, i_{2} \in[4] \wedge\left(i_{1} \neq i_{2}\right) \wedge\left(\left(\left(c_{i_{1}}, a_{i_{1}}\right),\left(c_{i_{2}}, a_{i_{2}}\right)\right),\left(\boldsymbol{x}, m, \mathrm{r}_{i}^{\text {enc }}\right)\right) \in \mathrm{R}_{\mathrm{IP}}^{2, \mathrm{ct}} \\ \wedge z_{0}=\operatorname{Com}\left(\left\{c_{i}\right\}_{i \in[4]} ; \mathrm{r}_{0}^{\text {com }}\right) \wedge z_{1}=\operatorname{Com}\left(0 ; \mathrm{r}_{1}^{\mathrm{com}}\right)\end{array}\right)$

- $\mathrm{R}^{\text {tok }}(x, w)=$ TRUE $\Longleftrightarrow \mathrm{P}_{1}^{\text {tok }}(x, w) \vee \mathrm{P}_{2}^{\text {tok }}(x, w)$, with, where

$$
\begin{aligned}
& \mathrm{P}_{1}^{\text {tok }}\left(\left(\boldsymbol{v},\left\{t_{i}\right\}_{i \in[4]},\left\{a_{i}\right\}_{i \in[4]}, z_{0}, z_{1}\right),\right. \\
& \left.\left(\left\{b_{i}\right\}_{i \in[4]},\left\{\mathrm{r}_{i}^{\mathrm{mpk}}\right\}_{i \in[4]},\left\{\mathrm{r}_{i}^{\text {tok }}\right\}_{i \in[4]}, i_{1}, i_{2}, i_{3}, \mathrm{r}_{0}^{\text {com }}, \mathrm{r}_{1}^{\text {com }}\right)\right)=\text { TRUE } \\
& \Longleftrightarrow\left(\begin{array}{c}
\forall i \in[4]:\left(\left(a_{i},\left(b_{i}, \mathrm{r}_{i}^{\mathrm{mpk}}\right)\right) \in \mathrm{R}^{\mathrm{mpk}} \wedge\right. \\
\left.\left(\left(a_{i}, t_{i}, \boldsymbol{v}_{i}\right),\left(b_{i}, \mathrm{r}_{i}^{\mathrm{mpk}}, \mathrm{r}_{i}^{\mathrm{tok}}\right)\right)\right) \in \mathrm{R}_{\mathrm{MP}}^{\mathrm{tok}} \\
\wedge z_{1}=\operatorname{Com}\left(1 ; \mathrm{r}_{1}^{\text {com }}\right)
\end{array}\right) \text {, and } \\
& \mathrm{P}_{2}^{\text {tok }}\left(\left(\boldsymbol{v},\left\{t_{i}\right\}_{i \in[4]},\left\{a_{i}\right\}_{i \in[4]}, z_{0}, z_{1}\right)\right. \text {, } \\
& \left.\left(\left\{b_{i}\right\}_{i \in[4]},\left\{\mathrm{r}_{i}^{\mathrm{mpk}}\right\}_{i \in[4]},\left\{\mathrm{r}_{i}^{\text {tok }}\right\}_{i \in[4]}, i_{1}, i_{2}, i_{3}, \mathrm{r}_{0}^{\text {com }}, \mathrm{r}_{1}^{\text {com }}\right)\right)=\text { TRUE } \\
& \Longleftrightarrow\left(\begin{array}{c}
i_{1}, i_{2}, i_{3} \in[4] \wedge\left(i_{1} \neq i_{2}\right) \wedge\left(i_{1} \neq i_{3}\right) \wedge\left(i_{2} \neq i_{3}\right) \\
\forall j \in[3]:\left(a_{i_{j}},\left(b_{i_{j}}, r_{i_{j}}^{\mathrm{mpk}}\right)\right) \in \mathrm{R}^{\mathrm{mpk}} \wedge \\
\left(\left(a_{i_{j}}, t_{i_{j}}, \boldsymbol{v}_{i_{j}}\right),\left(b_{i_{j}}, \mathrm{r}_{i_{j}}^{\mathrm{mpk}}, \mathrm{r}_{i_{j}}^{\text {tok }}\right)\right) \in \mathrm{R}_{\mathrm{IP}}^{\text {tok }} \\
\wedge z_{0}=\operatorname{Com}\left(\left\{c_{i}\right\}_{i \in[4]} ; \mathrm{r}_{1}^{\text {com }}\right) \wedge \\
\exists m \in \mathcal{M} \forall i \in[4] \operatorname{IP} . \operatorname{Dec}\left(c_{i}, t_{i}\right)=f_{\boldsymbol{v}}(m)
\end{array}\right)
\end{aligned}
$$

Construction 7 [Our VIPE VIP]

- VIP.SetUp $\left(1^{\lambda}, n\right) \rightarrow($ MPK, MSK $)$ :

1. For $i \in[4]$, run IP.SetUp $\left(1^{\lambda}, n\right)$ to generate $\left(\mathrm{MPK}_{i}, \mathrm{MSK}_{i}\right)$.

2. Run the commitment algorithm to generate $Z_{0}=\operatorname{Com}\left(0 ; r_{0}^{\text {com }}\right)$ and $\mathrm{Z}_{1}=\operatorname{Com}\left(1 ; \mathrm{r}_{1}^{\mathrm{com}}\right)$. 
3. Output VIP.MPK $=\left(\left\{\mathrm{MPK}_{i}\right\}_{i \in[4]}, \mathrm{Z}_{0}, \mathrm{Z}_{1}\right)$, VIP.MSK $=\left(\left\{\mathrm{MSK}_{i}\right\}_{i \in[4]}\right.$, $\left.r_{0}^{\text {com }}, r_{1}^{\text {com }}\right)$.

- VIP.Enc(MPK, $m, \boldsymbol{x}) \rightarrow$ CT:

1. For $i \in[4]$, run the encryption algorithm to compute $\mathrm{CT}_{i}=$ IP.Enc $\left(\mathrm{MPK}, m, \boldsymbol{x} ; \mathrm{r}_{i}^{\mathrm{enc}}\right)$.

2. Set $x=\left(\left\{\mathrm{CT}_{i}\right\}_{i \in[4]},\left\{\mathrm{MPK}_{i}\right\}_{i \in[4]}, \mathrm{Z}_{0}, \mathrm{Z}_{1}\right), w=\left(m, \boldsymbol{x},\left\{\mathrm{r}_{i}^{\text {enc }}\right\}_{i \in[4]}, 0,0\right.$, $\left.0^{\left|u_{0}\right|}, 0^{\left|u_{1}\right|}\right)$, and run $\mathcal{P}^{\text {enc }}(x, w)$ to generate $\pi_{\text {ct }}$ for relation $\mathrm{R}^{\text {enc }}(x, w)$. Note that $\mathrm{P}_{1}^{\text {enc }}(x, w)=$ TRUE.

3. Output ciphertext $\mathrm{CT}=\left(\left\{\mathrm{CT}_{i}\right\}_{i \in[4]}, \pi_{\mathrm{ct}}\right)$.

- VIP.TokGen(MPK, MSK, $\left.f_{\boldsymbol{v}}\right)$ :

1. For $i \in[4]$, run IP.TokGen(MSK, $\left.\boldsymbol{v} ; \mathrm{r}_{i}^{\text {tok }}\right)$ to generate Tok $_{\boldsymbol{v}}^{i}$.

2. $x=\left(\boldsymbol{v},\left\{\operatorname{Tok}_{\boldsymbol{v}}^{i}\right\}_{i \in[4]},\left\{\mathrm{MPK}_{i}\right\}_{i \in[4]}, \mathrm{Z}_{0}, \mathrm{Z}_{1}\right), w=\left(\left\{\mathrm{MSK}_{i}\right\}_{i \in[4]},\left\{\mathrm{rrk}_{i}^{\text {tok }}\right\}_{i \in[4]}\right.$, $\left.0,0,0,0^{\mid \text {rom }_{0}^{\text {com }} \mid},\left|\mathrm{r}_{1}^{\text {com }}\right|\right)$ run $\mathcal{P}^{\text {tok }}$ to generate $\pi_{\text {tok }}$ to prove $\mathrm{R}^{\text {tok }}(x, w)=$ TRUE. Note that $\mathrm{P}_{1}^{\text {tok }}(x, w)=$ TRUE

3. Output token $\operatorname{Tok}_{v}=\left(\left\{\operatorname{Tok}_{v}^{i}\right\}_{i \in[4]}, \pi_{\text {tok }}\right)$.

- VIP.Dec(MPK, $f_{v}$, Tok $_{v}$, CT):

1. Run the verification algorithms $\mathcal{V}^{\text {mpk }}, \mathcal{V}^{\text {enc }}, \mathcal{V}^{\text {tok }}$ on input the corresponding pairs of statement and proof (the proof for the verification of the master public key is set to the empty string). If some verification algorithms fails, then stop and output $\perp$ or go to the next step otherwise.

2. For all $i \in[4]$, compute $m^{(i)}=\operatorname{IP} . \operatorname{Dec}\left(\operatorname{Tok}_{\boldsymbol{v}}^{(i)}, \mathrm{CT}_{i}\right)$ and output the following:

$$
\left\{\begin{array}{l}
\text { If } \exists i_{1}, i_{2}, i_{3} \in[4] \text { s.t. } m=m^{\left(i_{1}\right)}=m^{\left(i_{2}\right)}=m^{\left(i_{3}\right)} \Rightarrow \text { Output } m . \\
\text { If } \nexists i_{1}, i_{2}, i_{3} \in[4] \text { s.t. } m^{\left(i_{1}\right)}=m^{\left(i_{2}\right)}=m^{\left(i_{3}\right)} \Rightarrow \text { Output } \perp .
\end{array}\right.
$$

- VIP.VrfyMPK(MPK): run $\mathcal{V}^{\mathrm{mpk}}(\mathrm{MPK}, \epsilon)$ and output its result.

- VIP.VrfyCT $\left.\left(\left(\left\{\mathrm{CT}_{i}\right\}_{i \in[4]},\left\{\mathrm{MPK}_{i}\right\}_{i \in[4]}, \mathrm{Z}_{0}, \mathrm{Z}_{1}\right), \pi_{\mathrm{ct}}\right)\right)$ : run $\mathcal{V}^{\text {enc }}\left(\left(\left\{\mathrm{CT}_{i}\right\}_{i \in[4]},\left\{\mathrm{MPK}_{i}\right\}_{i \in[4]}, \mathrm{Z}_{0}, \mathrm{Z}_{1}\right), \pi_{\mathrm{ct}}\right)$ and output its result.

- VIP.VrfyTok $\left(\left(\boldsymbol{v},\left\{\operatorname{Tok}_{\boldsymbol{v}}^{i}\right\}_{i \in[4]},\left\{\mathrm{MPK}_{i}\right\}_{i \in[4]}, \mathrm{Z}_{0}, \mathrm{Z}_{1}\right), \pi_{\text {tok }}\right)$ :

run $\mathcal{V}^{\text {tok }}\left(\left(\boldsymbol{v},\left\{\text { Tok }_{\boldsymbol{v}}^{i}\right\}_{i \in[4]},\left\{\mathrm{MPK}_{i}\right\}_{i \in[4]}, \mathbf{Z}_{0}, \mathbf{Z}_{1}\right), \pi_{\text {tok }}\right)$ and output its result.

Correctness of VIP follows from perfect correctness of IP. IND-Security and Verifiability of VIP follows as corollary (following Theorem 2) from the verifiability and IND-Security of the construction of [2] for general functions.

Theorem 2. If IP is a perfectly correct IND-Secure IP scheme for message space $\mathcal{M}$ and for the set $\mathbb{Z}_{p}^{n}$ of vectors of length $n$ over $\mathbb{Z}_{p}$, and $\mathrm{NIWI}{ }^{\mathrm{mpk}}, \mathrm{NIWI}^{\mathrm{ct}}, \mathrm{NIWI}^{\text {tok }}$ are NIWI systems resp. for the relations $R_{3}^{\mathrm{mpk}}, R_{3}^{\mathrm{enc}}, R_{3}^{\text {tok }}$ and Com is a noninteractive perfectly binding and computationally hiding commitment scheme, then VIP is an IND-Secure VIPE scheme for the class of inner product functionality over $\mathcal{M}$ and $\mathbb{Z}_{p}^{n}$. 


\section{NIWI Proofs and Verification Algorithms}

In this section we present the proof systems that we used in our VIP scheme, to prove membership of relations $R_{3}^{\text {mpk }}, R^{\text {tok }}$ and $R_{3}^{\text {enc }}$. For each of our relations ${ }^{2}$, we need to define a system of equations such that satisfiability of that system and the membership in the relation are equivalent. Then, the GS generic prover and verifier algorithms, $\mathrm{NIWI}_{\mathrm{GS}}=\left\langle\mathcal{P}_{\mathrm{GS}}, \mathcal{V}_{\mathrm{GS}}\right\rangle$, can be used for such equations. In this section, for each of our relations of Sect. 4, we will either define a corresponding system of equations or we will show how to implement directly (without using GS proofs).

Definition 6 (Pairing Product System of Equations). Consider a bilinear map $\mathbf{e}: \mathbb{G} \times \mathbb{G} \rightarrow \mathbb{G}_{T}$. The following system of equation with $k$ equations over $m$ variables $\mathcal{X}_{i} \in \mathbb{G}, i \in[m]$ and constants $B_{i}^{(t)} \in \mathbb{G}, \tau^{(t)} \in \mathbb{G}_{T}$ and $\gamma_{i j}^{(t)} \in \mathbb{Z}_{p}$ for $i \in[m], t \in[k]$ is called a pairing product system of equations over $\left(\mathbb{G}_{1}, \mathbb{G}_{T}, e\right)$ :

$$
\mathrm{E}:\left\{\begin{array}{l}
\prod_{i=1}^{m} \mathbf{e}\left(\mathcal{X}_{i}, B_{i}^{(1)}\right) \cdot \prod_{i=1}^{m} \prod_{j=1}^{m} \mathbf{e}\left(\mathcal{X}_{i}, \mathcal{X}_{j}\right)^{\gamma_{i j}^{(1)}}=\tau^{(1)} \\
\cdots \\
\prod_{i=1}^{m} \mathbf{e}\left(\mathcal{X}_{i}, B_{i}^{(k)}\right) \cdot \prod_{i=1}^{m} \prod_{j=1}^{m} \mathbf{e}\left(\mathcal{X}_{i}, \mathcal{X}_{j}\right)^{\gamma_{i j}^{(k)}}=\tau^{(k)}
\end{array}\right.
$$

$\left(g_{1}, g_{2}, \ldots, g_{m}\right) \in \mathbb{G}^{m}$ is a solution for the equation $\mathrm{E}$ iff

$$
\left(\mathrm{E}\left[\left(g_{1}, \ldots, g_{m}\right)\right]=\mathrm{TRUE}\right)=\left\{\begin{array}{l}
\prod_{i=1}^{m} \mathbf{e}\left(g_{i}, B_{i}^{(1)}\right) \cdot \prod_{i=1}^{m} \prod_{j=1}^{m} \mathbf{e}\left(g_{i}, g_{j}\right)^{\gamma_{i j}^{(1)}}=\tau^{(1)} \\
\ldots \\
\prod_{i=1}^{m} \mathbf{e}\left(g_{i}, B_{i}^{(k)}\right) \cdot \prod_{i=1}^{m} \prod_{j=1}^{m} \mathbf{e}\left(g_{i}, g_{j}\right)^{\gamma_{i j}^{(k)}}=\tau^{(k)}
\end{array}\right.
$$

We define the following relation for pairing product system of equations:

$$
R_{\boldsymbol{E}}=\left\{(x, w) \mid x=\mathrm{E}, w=\left(g_{1}, \ldots, g_{m}\right): \mathrm{E}\left[\left(g_{1}, \ldots, g_{m}\right)\right]=\mathrm{TRUE}\right\}
$$

Throughout the paper, we denote by $\mathrm{NIWI}_{\mathrm{GS}}=\left\langle\mathcal{P}_{\mathrm{GS}}, \mathcal{V}_{\mathrm{GS}}\right\rangle$ a Groth-Sahai [14] NIWI-proof system. Precisely:

$$
\text { - } \mathcal{P}_{\mathrm{GS}}\left(x=\mathrm{E}, w=\left(g_{1}, \ldots, g_{m}\right)\right) \rightarrow \pi_{\mathrm{E}} \bullet \mathcal{V}_{\mathrm{GS}}\left(x, \pi_{\mathrm{E}}\right) \rightarrow \begin{cases}1: & \text { If }(x, w) \in \mathrm{R}_{\mathrm{E}} \\ 0: & \text { Otherwise }\end{cases}
$$

\subsection{How to Handle Generalized or Statements}

Some of our relations of Sect. 4 consist of a generalized form of disjunction (OR) of two predicates, let us say $P_{1}$ and $P_{2}$. Suppose that we have equivalent systems

\footnotetext{
${ }^{2}$ Actually, we will implement some or part of them not directly using GS proofs.
} 
of equations for each of the two predicate, that is a system of equations $E_{1}$ (resp. $\left.E_{2}\right)$ representing predicate $P_{1}$ (resp. $P_{2}$ ). Consider the following relation:

$$
\begin{aligned}
\mathrm{R}_{\mathrm{OR}}= & \left\{(x, w) \mid x=\left(\mathrm{E}_{1}, \mathrm{E}_{2}\right), w=\left(\mathrm{idx}, w_{1}, w_{2}\right): \mathrm{idx} \in\{1,2\} \wedge\right. \\
& \left.\left(\mathrm{E}_{\mathrm{idx}}, w_{\mathrm{idx}}\right) \in \mathrm{R}_{\mathrm{E}} \wedge w_{\mathrm{idx}} \in \mathbb{G}^{3}\right\},
\end{aligned}
$$

where idx means $\{1,2\} /\{\mathrm{id} x\}$.

Notice that the relation is not exactly a disjunction of pairing product equations because we need to make sure that the statement that holds is the one selected by the index in the witness, so we cannot use the technique of Groth [12] and we will follow a different approach.

By hypothesis $\mathcal{P}_{\mathrm{GS}}$ takes as input a system of equations $\mathrm{E}$ as statement and a solution $\left(g_{1}, \ldots, g_{m}\right)$ as witness and provides a NIWI-proof of membership of $(\mathrm{E}, w) \in \mathrm{R}_{\mathrm{E}}$. Therefore, to use $\mathrm{NIWI}_{\mathrm{GS}}$ to generate a NIWI-proof for relation $\mathrm{R}_{\mathrm{OR}}$, we need to define a third system of equation $\mathrm{E}_{\mathrm{OR}}$ with the following properties:

1. $\mathrm{E}_{\mathrm{OR}} \approx \mathrm{R}_{\mathrm{OR}}$. With this notation, we mean that there exist two efficiently computable functions $f$ and $g$ such that:

$$
\begin{gathered}
\exists w=\left(\mathrm{idx}, w_{1}, w_{2}\right)\left(x=\left(\mathrm{E}_{1}, \mathrm{E}_{2}\right), w\right) \in \mathrm{R}_{\mathrm{OR}} \Leftrightarrow \exists \tilde{w}\left(\mathrm{E}_{\mathrm{OR}}=f(x), \tilde{w}\right) \in \mathrm{R}_{\mathrm{E}} . \\
(x, w) \in \mathrm{R}_{\mathrm{OR}} \Rightarrow(f(x), g(x, w)) \in \mathrm{R}_{\mathrm{OR}} .
\end{gathered}
$$

The latter properties guarantee that a proof for relation $\mathrm{R}_{\mathrm{OR}}$ computed using NIWI $I_{\text {GS }}$ satisfies completeness and soundness. For WI to hold, we need the following property.

2. The function $f$ is efficiently invertible.

Now we show how to construct the system of equations $E_{0 R}$ with the aforementioned properties. Consider two systems of pairing product equations $E_{1}$ and $E_{2}$ - same structure as in 1. For simplicity, we assume the equations are over two variables (the general case is straightforward).

$$
\mathrm{E}_{1}: \mathbf{e}\left(\mathcal{X}_{1}, a_{1}\right) \cdot \mathbf{e}\left(\mathcal{X}_{2}, a_{2}\right)=\tau_{1}, \mathrm{E}_{2}: \mathbf{e}\left(\mathcal{Y}_{1}, b_{1}\right) \cdot \mathbf{e}\left(\mathcal{Y}_{2}, b_{2}\right)=\tau_{2}
$$

We define the new system of equations $E_{O R}$ with 4 new variables $\mathcal{Z}_{11}, \mathcal{Z}_{12}$, $\mathcal{Z}_{21}, \mathcal{Z}_{22}$ as follows:

$$
\mathrm{E}_{\mathrm{OR}}:\left\{\begin{array}{l}
\mathbf{e}\left(\mathcal{X}_{1}, a_{1}\right) \cdot \mathbf{e}\left(\mathcal{X}_{2}, a_{2}\right) \cdot \mathbf{e}\left(\mathcal{Z}_{11}, \mathcal{Z}_{12}\right)=\tau_{1} \\
\mathbf{e}\left(\mathcal{Y}_{1}, b_{1}\right) \cdot \mathbf{e}\left(\mathcal{Y}_{2}, b_{2}\right) \cdot \mathbf{e}\left(\mathcal{Z}_{21}, \mathcal{Z}_{22}\right)=\tau_{2} \\
\mathbf{e}\left(\mathcal{Z}_{11}, \mathcal{Z}_{22}\right)=1 \\
\mathbf{e}\left(\mathcal{Z}_{11}, g\right) \cdot \mathbf{e}\left(\mathcal{Z}_{\mathrm{idx}}, g\right)=\mathbf{e}(g, g) \\
\mathbf{e}\left(\mathcal{Z}_{22}, g\right) \cdot \mathbf{e}\left(\mathcal{Z}_{\mathrm{idx}}, g\right)=\mathbf{e}\left(g^{2}, g\right)
\end{array}\right.
$$

Analysis of the Equations: Consider $\left(\mathcal{Z}_{\mathrm{idx}} \hookleftarrow g_{\mathrm{idx}}, \mathcal{X}_{1} \hookleftarrow g_{1}, \mathcal{X}_{2} \hookleftarrow g_{2}, \mathcal{Y}_{1} \hookleftarrow\right.$ $\left.g_{3}, \mathcal{Y}_{2} \hookleftarrow g_{4}, \mathcal{Z}_{11} \hookleftarrow g_{11}, \ldots, \mathcal{Z}_{22} \hookleftarrow g_{22}\right)$ as a solution for $\mathrm{E}_{\mathrm{OR}}$. So, there exist 
values idx, $z_{11}, z_{22} \in \mathbb{Z}_{p}$ such that $g_{\text {idx }}=g^{\text {idx }}, g_{11}=g^{z_{11}}, g_{22}=g^{z_{22}}$ and for $t \in[k]$ there exist values $\alpha_{t}$ such that $\tau_{t}=\mathbf{e}\left(g, \alpha_{t}\right)$.

- $\mathbf{e}\left(\mathcal{Z}_{11}, g\right) \cdot \mathbf{e}\left(\mathcal{Z}_{\mathrm{idx}}, g\right)=\mathbf{e}(g, g) \Rightarrow \mathbf{e}\left(g^{z_{11}+\mathrm{idx}-1}, g\right)=1$

$\Rightarrow z_{11}=1-\mathrm{idx}$ and similarly $z_{22}=2-\mathrm{idx}$.

- $\mathbf{e}\left(\mathcal{Z}_{11}, \mathcal{Z}_{22}\right)=1 \Rightarrow\left(z_{11}=0 \vee z_{22}=0\right)$

- $z_{11}=0 \wedge z_{11}=1-\mathrm{idx} \Rightarrow \mathbf{e}\left(\mathcal{X}_{1} \hookleftarrow g_{1}, a_{1}\right) \cdot \mathbf{e}\left(\mathcal{X}_{2} \hookleftarrow g_{2}, a_{2}\right)=\tau_{1}$

$\Rightarrow\left(\mathrm{E}_{1}\left[g_{1}, g_{2}\right]=\mathrm{TRUE} \wedge \mathrm{idx}=1\right)$

- Similarly, $z_{22}=0 \wedge z_{22}=2-\mathrm{idx}$

$$
\Rightarrow \mathbf{e}\left(\mathcal{Z}_{21}, \mathcal{Z}_{22}\right)=1 \Rightarrow\left(\mathrm{E}_{2}\left[g_{3}, g_{4}\right]=\operatorname{TRUE} \wedge \mathrm{idx}=2\right)
$$

The above facts imply that:

$\mathrm{E}_{\mathrm{OR}}\left[\left(g_{\mathrm{idx}}, g_{1}, \ldots, g_{4}, g_{11}, \ldots, g_{22}\right)\right]=$ TRUE $\Rightarrow$

$\left(\left(\mathrm{E}_{1}\left[g_{1}, g_{2}, \alpha_{1}\right]=\operatorname{TRUE} \wedge \mathrm{idx}=1\right) \vee\left(\mathrm{E}_{2}\left[g_{3}, g_{4}, \alpha_{2}\right]=\operatorname{TRUE} \wedge \mathrm{idx}=2\right)\right)$,

as it was to show. It is also easy to see that the previous transformation is efficiently invertible.

For the other direction, suppose w.l.o.g that $w_{1}=\left(g_{1}, g_{2}, \alpha_{1}\right)$ is a solution to $x=\mathrm{E}_{1}$ (the other case is symmetrical and we omit it), namely $\left(x, w_{1}\right) \in \mathrm{R}^{\prime}$ '. Suppose also that $w_{2}=\left(g_{3}, g_{4}, \alpha_{2}\right) \in \mathbb{G}^{3}$ is an arbitrary triple of elements of $\mathbb{G}$. Therefore $\left(1, w_{1}, w_{2}\right)$ is a witness to $\left(\mathrm{E}_{1}, \mathrm{E}_{2}\right)$ with respect to relation $\mathrm{R}_{\mathrm{OR}}$. Then, setting $\left(\mathcal{Z}_{\text {idx }} \hookleftarrow g^{1}, \mathcal{X}_{1} \hookleftarrow g_{1}, \mathcal{X}_{2} \hookleftarrow g_{2}, \mathcal{Y}_{1} \hookleftarrow g^{0}, \mathcal{Y}_{2} \hookleftarrow g^{0}, \mathcal{Z}_{11} \hookleftarrow g^{0}, \mathcal{Z}_{12} \hookleftarrow\right.$ $\left.g^{1}, \mathcal{Z}_{21} \hookleftarrow \alpha_{2}, \mathcal{Z}_{22} \hookleftarrow g^{1}\right)$, we have that:

$$
\mathrm{E}_{\mathrm{OR}}\left[\left(g_{\mathrm{idx}}, g_{1}, \ldots, g_{4}, g_{11}, \ldots, g_{22}\right)\right]=\text { TRUE. }
$$

(Notice that we implicitly defined a transformation $g$ as needed.)

\subsection{OR Proof in the General Case}

If the number of pairing products $(m)$ in each of the two equations is greater than 1 , such as:

$$
\mathrm{E}_{1}:\left\{\begin{array}{l}
\mathbf{e}\left(\mathcal{X}_{1}, a_{1}\right) \cdot \mathbf{e}\left(\mathcal{X}_{2}, a_{2}\right)=\tau_{1} \\
\mathbf{e}\left(\mathcal{X}_{1}, a_{1}^{\prime}\right) \cdot \mathbf{e}\left(\mathcal{X}_{2}, a_{2}^{\prime}\right)=\tau_{1}^{\prime}
\end{array} \quad, \mathrm{E}_{2}:\left\{\begin{array}{l}
\mathbf{e}\left(\mathcal{Y}_{1}, b_{1}\right) \cdot \mathbf{e}\left(\mathcal{Y}_{2}, b_{2}\right)=\tau_{2} \\
\mathbf{e}\left(\mathcal{Y}_{1}, b_{1}^{\prime}\right) \cdot \mathbf{e}\left(\mathcal{Y}_{2}, a_{2}^{\prime}\right)=\tau_{2}^{\prime}
\end{array}\right.\right.
$$

then $E_{O R}$ can be defined as:

$$
\mathrm{E}_{\mathrm{OR}}:\left\{\begin{array}{l}
\mathbf{e}\left(\mathcal{X}_{1}, a_{1}\right) \cdot \mathbf{e}\left(\mathcal{X}_{1}, a_{2}\right) \cdot \mathbf{e}\left(\mathcal{Z}_{11}, \mathcal{Z}_{12}\right)=\tau_{1} \\
\mathbf{e}\left(\mathcal{X}_{1}, a_{1}^{\prime}\right) \cdot \mathbf{e}\left(\mathcal{X}_{2}, a_{2}^{\prime}\right) \cdot \mathbf{e}\left(\mathcal{Z}_{11}, \mathcal{Z}_{13}\right)=\tau_{1}^{\prime} \\
\mathbf{e}\left(\mathcal{Y}_{1}, b_{1}\right) \cdot \mathbf{e}\left(\mathcal{Y}_{2}, b_{2}\right) \cdot \mathbf{e}\left(\mathcal{Z}_{21}, \mathcal{Z}_{22}\right)=\tau_{2} \\
\mathbf{e}\left(\mathcal{Y}_{1}, b_{1}^{\prime}\right) \cdot \mathbf{e}\left(\mathcal{Y}_{2}, b_{2}^{\prime}\right) \cdot \mathbf{e}\left(\mathcal{Z}_{23}, \mathcal{Z}_{22}\right)=\tau_{2}^{\prime} \\
\mathbf{e}\left(\mathcal{Z}_{11}, \mathcal{Z}_{22}\right)=1 \\
\mathbf{e}\left(\mathcal{Z}_{11}, g\right) \cdot \mathbf{e}\left(\mathcal{Z}_{\text {idx }}, g\right)=\mathbf{e}(g, g) \\
\mathbf{e}\left(\mathcal{Z}_{22}, g\right) \cdot \mathbf{e}\left(\mathcal{Z}_{\text {idx }}, g\right)=\mathbf{e}\left(g^{2}, g\right)
\end{array}\right.
$$

We omit further details. 
Notations: For the rest of this section, let us fix $n \in \mathbb{N}$ as dimension of the vector space and let $i \in[n], b \in[2]$. Note we can efficiently check whether a string is a valid group element. We recall what follows.

$$
\begin{aligned}
& \text { mpk }=\left(g, h,\left\{W_{b, i}, F_{b, i}, T_{b, i}, H_{b, i}, U_{b}, V_{b}\right\}, K_{1}, K_{2}, \Lambda\right) \in \mathbb{G}^{4 n+8} \times \mathbb{G}_{T} \\
& \text { msk }=\left(\left\{w_{b, i}, f_{b, i}, t_{b, i}, h_{b, i}, \delta_{b}, \theta_{b}\right\}, \Omega, k\right) \in \mathbb{Z}_{p}^{4 n+6} \\
& \text { tok }=\left(K_{A}, K_{B},\left\{K_{3, i}, K_{4, i}, K_{5, i}, K_{6, i}\right\}\right) \in \mathbb{G}^{4 n+2} \\
& \mathrm{ct}=\left(\left(\mathrm{ct}_{1}, \mathrm{ct}_{2},\left\{\begin{array}{l}
\mathrm{ct}_{3, i}, \mathrm{ct}_{4, i} \\
\mathrm{ct}_{5, i}, \mathrm{ct}_{6, i}
\end{array}\right\}, \mathrm{ct}_{7}, \mathrm{ct}_{8}\right),\right. \\
&\left.\qquad\left(\mathrm{ct}_{1}^{\prime}, \mathrm{ct}_{2}^{\prime},\left\{\begin{array}{l}
\mathrm{ct}_{3, i}^{\prime}, \mathrm{ct}_{4, i}^{\prime} \\
\mathrm{ct}_{5, i}^{\prime}, \mathrm{ct}_{6, i}^{\prime}
\end{array}\right\}, \mathrm{ct}_{7}^{\prime}, \mathrm{ct}_{8}^{\prime}\right)\right) \in \mathbb{G}^{8 n+6} \times \mathbb{G}_{T}^{2}
\end{aligned}
$$

\subsection{Master Public Key Verification}

Let $x=$ mpk. Since $g$ and $\mathbf{e}(g, g)$ are generators for the groups $\mathbb{G}$ and $\mathbb{G}_{T}$ of prime order $p$, we can represent all components of $x$ as a power of either $g$ or $\mathbf{e}(g, g)$. That is, there exist $\Omega, k^{\prime},\left\{w_{b, i}, f_{b, i}, t_{b, i}, h_{b, i}\right\},\left\{\delta_{b}, \theta_{b}, k_{b}\right\}$ for $i \in[n]$ and $b \in[2]$, in $\mathbb{Z}_{p}$ such that: $h=g^{\Omega}, \Lambda=\mathbf{e}(g, g)^{k^{\prime}}, W_{b, i}=g^{w_{b, i}, F_{b, i}}=g^{f_{b, i}}, T_{b, i}=$ $g^{t_{b, i}}, H_{b, i}=g^{h_{b, i}}, U_{b}=g^{\delta_{b}}, V_{b}=g^{\theta_{b}}, K_{b}=g^{k_{b}}$. The following holds:

$$
\begin{aligned}
& \mathbf{e}(g, h)=\mathbf{e}\left(U_{1}, W_{2, i}\right) \cdot \mathbf{e}\left(U_{2}, W_{1, i}\right)^{-1}=\mathbf{e}\left(V_{1}, T_{2, i}\right) \cdot \mathbf{e}\left(V_{2}, T_{1, i}\right)^{-1} \Rightarrow \\
& \mathbf{e}\left(g, g^{\Omega}\right)=\mathbf{e}\left(g^{\delta_{1}}, g^{w_{2, i}}\right) \cdot \mathbf{e}\left(g^{\delta_{2}}, g^{-w_{1, i}}\right)=\mathbf{e}\left(g^{\theta_{1}}, g^{t_{2, i}}\right) \cdot \mathbf{e}\left(g^{\theta_{2}}, g^{-t_{1, i}}\right) \\
& \Rightarrow \Omega=\delta_{1} w_{2, i}-\delta_{2} w_{1, i}=\theta_{1} t_{2, i}-\theta_{2} t_{1, i} . \\
& \mathbf{e}\left(K_{1}, K_{2}\right)=\mathbf{e}\left(g^{k_{1}}, g^{k_{2}}\right)=\Lambda=\mathbf{e}\left(g, g^{k^{\prime}}\right) \Rightarrow k^{\prime}=k_{1} k_{2}
\end{aligned}
$$

By defining $g^{\prime}=g^{k^{\prime}}, K_{1}=g^{k_{1}}, K_{2}=g^{k_{2}}$, it follows that:

$$
\Lambda=e\left(K_{1}, K_{2}\right), K_{1}=g^{k}, K_{2}=g^{\prime \frac{1}{k}}
$$

Hence, we have the verification algorithm in Fig. 2 for master public key.

Input: mpk, Output: 1 if mpk is a well-generated master public key for IP scheme and 0 otherwise

(1) If $\Lambda \neq \mathbf{e}\left(K_{1}, K_{2}\right)$. output 0 otherwise go to the next step

(2) For $i=1$ to $n$ do :

(i.a) If $\mathbf{e}\left(U_{1}, W_{2, i}\right) \cdot \mathbf{e}\left(U_{2}, W_{1, i}\right)^{-1} \neq \mathbf{e}(h, g)$ output 0 else go to the next step

(i.b) If $\mathbf{e}\left(V_{1}, T_{2, i}\right) \cdot \mathbf{e}\left(V_{2}, T_{1, i}\right)^{-1} \neq \mathbf{e}(h, g)$ output 0 else go to the next step

(3) Output 1.

Fig. 2. Master public key verification algorithm. (membership in relation $\mathrm{R}_{3}^{\mathrm{mpk}}$ ) 


\subsection{Token Verification Algorithms}

As it was defined in Sect. 4 , there are two relations for tokens, $R_{3}^{\text {tok }}$ and $R^{\text {tok }}$. The algorithm in Fig. 3 verifies membership in relation $\mathrm{R}_{\mathrm{IP}}^{\text {tok }}$.

\section{Input: $\mathrm{MPK}, \boldsymbol{v}=\left(v_{1}, \ldots, v_{n}\right) \neq \mathbf{0}$, tok}

Output: 1 if tok is a well-generated token for IP scheme and 0 otherwise

1. If $\boldsymbol{v}=\mathbf{0}$ output 0 else let $i^{*}$ be an index such that $v_{i *} \neq 0$

2. Compute $\Lambda_{1}^{*}=\mathbf{e}\left(K_{3, i}, U_{1}\right) \cdot \mathbf{e}\left(K_{4, i}, U_{2}\right)$ and $\Lambda_{2}^{*}=\mathbf{e}\left(K_{5, i}, V_{1}\right) \cdot \mathbf{e}\left(K_{6, i}, V_{2}\right)$

3. If $\Lambda_{1}^{*}=1_{\mathbb{G}_{T}}$ OR $\Lambda_{2}^{*}=1_{\mathbb{G}_{T}}$ output $\perp$

4. For $i=1$ to $n$ do:
(a) If $\left(\mathbf{e}\left(K_{3, i}, U_{1}\right) \cdot \mathbf{e}\left(K_{4, i}, U_{2}\right)\right)^{v_{i^{*}}} \neq\left(\Lambda_{1}^{*}\right)^{v_{i}}$ output 0
(b) If $\left(\mathbf{e}\left(K_{5, i}, V_{1}\right) \cdot \mathbf{e}\left(K_{6, i}, V_{2}\right)\right)^{v_{i^{*}}} \neq\left(\Lambda_{2}^{*}\right)^{v_{i}}$ output 0

5. If $\Lambda \prod_{i=1}^{n} \mathbf{e}\left(K_{3, i}, F_{1, i}\right)^{-1} \cdot \mathbf{e}\left(K_{4, i}, F_{2, i}\right)^{-1} \cdot \mathbf{e}\left(K_{5, i}, H_{1, i}\right)^{-1} \mathbf{e}\left(K_{6, i}, H_{2, i}\right)^{-1} \neq$ $\mathbf{e}\left(K_{A}, g\right)$ output 0 .

6. If $\prod_{i=1}^{n} \mathbf{e}\left(K_{3, i}, W_{1, i}\right) \cdot \mathbf{e}\left(K_{4, i}, W_{2, i}\right) \cdot \mathbf{e}\left(K_{5, i}, T_{1, i}\right) \cdot \mathbf{e}\left(K_{6, i}, T_{2, i}\right) \neq \mathbf{e}\left(h, K_{B}\right)^{-1}$ output 0 .

7. Output 1.

Fig. 3. First token verification algorithm. (membership in relation $\mathrm{R}_{\mathrm{IP}}^{\text {tok }}$ )

Correctness of the algorithm: For simplicity let's assume $v_{1} \neq 0$ and $i^{*}=1$.

- $\Lambda_{1}^{*}, \Lambda_{2}^{*} \in \mathbb{G}_{T} \Rightarrow \exists \lambda_{1}, \lambda_{2} \in \mathbb{Z}_{p}$ s.t. $\Lambda_{1}^{*}=e(g, h)^{\lambda_{1} v_{1}}, \Lambda_{2}^{*}=e(g, h)^{\lambda_{2} v_{1}}$

- $\forall i \in[n] \exists r_{i}, r_{i}^{\prime} \in \mathbb{Z}_{p}$ s.t. $K_{3, i}=g^{-\delta_{2} r_{i}} \cdot g^{\lambda_{1} v_{i} w_{2, i}}, K_{4, i}=g^{\delta_{1} r_{i}^{\prime}} \cdot g^{-\lambda_{1} v_{i} w_{1, i}}$

$$
\begin{aligned}
& \Rightarrow \mathbf{e}\left(K_{3, i}, U_{1}\right) \cdot \mathbf{e}\left(K_{4, i}, U_{2}\right)=\mathbf{e}\left(g^{-\delta_{2} r_{i}} \cdot g^{\lambda_{1} v_{i} w_{2, i}}, g^{\delta_{1}}\right) \cdot \mathbf{e}\left(g^{\delta_{1} r_{i}^{\prime}} \cdot g^{-\lambda_{1} v_{i} w_{1, i}}, g^{\delta_{2}}\right)= \\
& \quad \mathbf{e}(g, g)^{\delta_{1} \delta_{2}\left(r_{i}^{\prime}-r_{i}\right)} \cdot \mathbf{e}(g, h)^{\lambda_{1} v_{i}}= \\
& \Rightarrow\left(\mathbf{e}\left(K_{3, i}, U_{1}\right) \cdot \mathbf{e}\left(K_{4, i}, U_{2}\right)\right)^{v_{1}}=\mathbf{e}(g, g)^{v_{1} \delta_{1} \delta_{2}\left(r_{i}^{\prime}-r_{i}\right)} \cdot \mathbf{e}(g, h)^{\lambda_{1} v_{1} v_{i}}
\end{aligned}
$$

- Step 3: $\Lambda_{1}^{*} \neq 1_{\mathbb{G}_{T}}, \Lambda_{2}^{*} \neq 1_{\mathbb{G}_{T}} \Rightarrow \lambda_{1} \neq 0, \lambda_{2} \neq 0$

- Step 4.a: If $\left(\mathbf{e}\left(K_{3, i}, U_{1}\right) \cdot \mathbf{e}\left(K_{4, i}, U_{2}\right)\right)^{v_{1}}=\left(\Lambda_{1}^{*}\right)^{v_{i}} \Rightarrow \mathbf{e}(g, g)^{v_{1} \delta_{1} \delta_{2}\left(r_{i}^{\prime}-r_{i}\right)}$. $\mathbf{e}(h, g)^{\lambda_{1} v_{1} v_{i}}=\mathbf{e}(g, h)^{\lambda_{1} v_{1} v_{i}} \Rightarrow \mathbf{e}(g, g)^{v_{1} \delta_{1} \delta_{2}\left(r_{i}^{\prime}-r_{i}\right)}=1_{\mathbb{G}_{T}} \Rightarrow \forall i \in[n]: r_{i}=$ $r_{i}^{\prime} \Rightarrow K_{3, i}=g^{-\delta_{2} r_{i}} \cdot g^{\lambda_{1} v_{i} w_{2, i}}, K_{4, i}=g^{\delta_{1} r_{i}} \cdot g^{-\lambda_{1} v_{i} w_{1, i}}$ And similar computations show that the equality in step (4.b) holds for all $i \in[n]$. Then we conclude that there exists $\phi_{i} \in \mathbb{Z}_{p}$ such that: $K_{5, i}=g^{-\theta_{2} \phi_{i}} \cdot g^{\lambda_{2} v_{i} t_{2, i}}, K_{6, i}=$ $g^{\theta_{1} \phi_{i}} \cdot g^{-\lambda_{2} v_{i} t_{1, i}}$. 
- Step 5

$$
\begin{aligned}
& K_{A}=g^{\prime} \prod_{i=1}^{n} K_{3, i}^{-f_{1, i}} K_{4, i}^{-f_{2, i}} K_{5, i}^{-h_{1, i}} K_{6, i}^{-h_{2, i}} \\
\Longleftrightarrow & \mathbf{e}\left(K_{A}, g\right)=\mathbf{e}\left(g^{\prime} \prod_{i=1}^{n} K_{3, i}^{-f_{1, i}} K_{4, i}^{-f_{2, i}} K_{5, i}^{-h_{1, i}} K_{6, i}^{-h_{2, i}}, g\right) \\
\Longleftrightarrow & \mathbf{e}\left(K_{A}, g\right)=\Lambda \cdot \prod_{i=1}^{n} \mathbf{e}\left(K_{3, i}, F_{1, i}\right)^{-1} \cdot \mathbf{e}\left(K_{4, i}, F_{2, i}\right)^{-1} \cdot \mathbf{e}\left(K_{5, i}, H_{1, i}\right)^{-1} \\
& \mathbf{e}\left(K_{6, i}, H_{2, i}\right)^{-1} .
\end{aligned}
$$

\section{- Step 6}

$$
\begin{aligned}
& \prod_{i=1}^{n} \mathbf{e}\left(K_{3, i}, W_{1, i}\right) \cdot \mathbf{e}\left(K_{4, i}, W_{2, i}\right) \cdot \mathbf{e}\left(K_{5, i}, T_{1, i}\right) \cdot \mathbf{e}\left(K_{6, i}, T_{2, i}\right)=\mathbf{e}\left(h, K_{B}\right)^{-1} \\
= & \prod_{i=1}^{n} \mathbf{e}\left(g^{r_{i}\left(\delta_{1} w_{2, i}-\delta_{2} w_{1, i}\right)}, g\right) \cdot \mathbf{e}\left(g^{\phi_{i}\left(\theta_{1} t_{2, i}-\theta_{2} t_{1, i}\right)}, g\right)=\mathbf{e}\left(h, K_{B}\right)^{-1} \\
= & \prod_{i=1}^{n} \mathbf{e}(g, h)^{r_{i}+\phi_{i}}=\mathbf{e}\left(h, K_{B}\right)^{-1} \Rightarrow K_{B}=\prod_{i=1}^{n} g^{-\left(r_{i}+\phi_{i}\right)}
\end{aligned}
$$

The second relation is a disjunction of two predicates, $\mathrm{R}^{\text {tok }}(x, w)=P_{1}^{\text {tok }} \vee P_{2}^{\text {tok }}$. The proof of membership for this relation can be implemented using the equations for the token verification algorithm for relation $\mathrm{R}_{3}^{\text {tok }}$ Fig. 3 and assuming to have pairing product equations corresponding to the commitments in the two aforementioned predicates. We skip further details.

\subsection{NIWI ${ }^{\text {enc }}=\left\langle\mathcal{P}^{\text {enc }}, \mathcal{V}^{\text {enc }}\right\rangle$ : NIWI-Proof for Encryption Algorithm}

For the relation $\mathrm{R}_{M \mathrm{P}}^{\mathrm{ct}}$, we first provide a proof of satisfiability for a system of equations related to a single ciphertext, that is $k=1$, and we will later extend it to the case of two ciphertexts, that is $k=2$. For $k>2$, the algorithm is similar to the case $k=2$.

Let $x=(\mathrm{mpk}, \mathrm{ct})$. We define the following variables for $i \in[n]$ :

$$
\begin{aligned}
& \mathcal{S}_{1}=g^{s_{1}}, \mathcal{S}_{3}=g^{s_{3}}, \mathcal{S}_{4}=g^{s_{4}}, \mathcal{X}_{i}=g^{x_{i}}, \mathcal{S}_{1}^{\prime}=g^{s_{1}^{\prime}}, \mathcal{S}_{3}^{\prime}=g^{s_{3}^{\prime}}, \mathcal{U}_{1}=U_{1}^{s_{3}}, \\
& \mathcal{U}_{2}=U_{2}^{s_{3}}, \mathcal{V}_{1}=V_{1}^{s_{4}}, \mathcal{V}_{2}=V_{2}^{s_{4}}, \mathcal{U}_{1}^{\prime}=U_{1}^{s_{3}^{\prime}}, \mathcal{U}_{2}^{\prime}=U_{2}^{s_{3}^{\prime}}, \mathcal{K}_{1}=K_{1}^{s_{2}}, \mathcal{K}_{1}^{\prime}=K_{1}^{s_{2}^{\prime}}
\end{aligned}
$$

We have the following Equations related to component $\mathrm{ct}_{2}\left(\mathrm{ct}_{2}^{\prime}\right)$ :

$$
\mathbf{e}\left(\mathrm{ct}_{2}, g\right)=\mathbf{e}\left(h^{s_{1}}, g\right)=\mathbf{e}\left(h, g^{s_{1}}\right)=\mathbf{e}\left(h, \mathcal{S}_{1}\right),\left(\mathbf{e}\left(\mathrm{ct}_{2}^{\prime}, g\right)=\mathbf{e}\left(h, \mathcal{S}_{1}^{\prime}\right)\right)
$$


and related equation to $\mathrm{ct}_{3, i}$ for $i \in[n]$ : (Same computation results the same equations for $\mathrm{ct}_{j, i}, \mathrm{ct}_{j, i}^{\prime}$ for $\left.j=3,4,5,6\right)$

$$
\begin{aligned}
\mathbf{e}\left(\mathrm{ct}_{3, i}, g\right) & =\mathbf{e}\left(W_{1, i}^{s_{1}}, g\right) \cdot \mathbf{e}\left(F_{1, i}^{s_{2}}, g\right) \cdot \mathbf{e}\left(U_{1}^{s_{3} x_{i}}, g\right) \\
& =\mathbf{e}\left(W_{1, i}, g^{s_{1}}\right) \cdot \mathbf{e}\left(F_{1, i}, g^{s_{2}}\right) \cdot \mathbf{e}\left(U_{1}^{s_{3}}, g^{x_{i}}\right) \\
& \left.=\mathbf{e}\left(W_{1, i}, \mathcal{S}_{1}\right) \cdot \mathbf{e}\left(F_{1, i}, \mathrm{ct}\right)_{1}\right) \cdot \mathbf{e}\left(\mathcal{U}_{1}, \mathcal{X}_{i}\right) \\
\Rightarrow & \mathbf{e}\left(\mathrm{ct}_{3, i}, g\right) \cdot \mathbf{e}\left(F_{1, i}, \mathrm{ct}_{1}\right)^{-1}=\mathbf{e}\left(W_{1, i}, \mathcal{S}_{1}\right) \cdot \mathbf{e}\left(\mathcal{U}_{1}, \mathcal{X}_{i}\right)
\end{aligned}
$$

The equations show that the exponent of $U_{b}^{s_{3}}$ and $V_{b}^{s_{4}}$ in $\mathrm{ct}_{3, i}, \mathrm{ct}_{4, i}, \mathrm{ct}_{5, i}, \mathrm{ct}_{6, i}$ are $x_{i}$. So we have the following equation:

$$
\begin{gathered}
\mathbf{e}\left(\mathcal{U}_{1}, U_{2}\right) \cdot \mathbf{e}\left(U_{1}^{-1}, \mathcal{U}_{2}\right)=\mathbf{e}\left(U^{s_{3}}, U_{2}\right) \cdot \mathbf{e}\left(U_{1}^{-1}, U_{2}^{s_{3}}\right)=\mathbf{e}\left(U_{1}, U_{2}\right)^{s_{3}-s_{3}}=1_{\mathbb{G}_{T}} \\
\mathbf{e}\left(\mathcal{V}_{1}, V_{2}\right) \cdot \mathbf{e}\left(V_{1}^{-1}, \mathcal{V}_{2}\right)=\mathbf{e}\left(V^{s_{4}}, V_{2}\right) \cdot \mathbf{e}\left(V_{1}^{-1}, V_{2}^{s_{4}}\right)=\mathbf{e}\left(V_{1}, V_{2}\right)^{s_{4}-s_{4}}=1_{\mathbb{G}_{T}}
\end{gathered}
$$

The equation related to $\mathrm{ct}_{7}=\mathbf{e}\left(g^{s_{3}}, g^{s_{4}}\right)$ is the following:

$$
\mathrm{ct}_{7}=\mathbf{e}\left(g^{s_{3}}, g^{s_{4}}\right)=\mathbf{e}\left(\mathcal{S}_{3}, \mathcal{S}_{4}\right), \mathrm{ct}_{7}^{\prime}=\mathbf{e}\left(g^{s_{3}^{\prime}}, g^{s_{4}}\right)=\mathbf{e}\left(\mathcal{S}_{3}^{\prime}, \mathcal{S}_{4}\right)
$$

To prove $s_{3} \neq s_{3}^{\prime}$, we just need to check whether $\mathrm{ct}_{7} \neq \mathrm{ct}_{7}^{\prime}$ or not.

$$
\mathrm{ct}_{7} \neq \mathrm{ct}_{7}^{\prime} \Rightarrow \mathbf{e}\left(g^{s_{3}}, g^{s_{4}}\right) \neq \mathbf{e}\left(g^{s_{3}^{\prime}}, g^{s_{4}}\right) \Rightarrow s_{3} \neq s_{3}^{\prime} \text {. }
$$

The equation related to $\mathrm{ct}_{8}, \mathrm{ct}_{8}^{\prime}$ is the following:

$$
\begin{aligned}
& \mathrm{ct}_{8}=\Lambda^{-s_{2}} \cdot m, \mathrm{ct}_{8}^{\prime}=\Lambda^{-s_{2}^{\prime}} \cdot m \Rightarrow \mathrm{ct}_{8}^{-1} \cdot \mathrm{ct}_{8}^{\prime}=\Lambda^{s_{2}} \cdot m^{-1} \Lambda^{-s_{2}^{\prime}} \cdot m=\Lambda^{s_{2}-s_{2}^{\prime}} \\
\Rightarrow & \mathrm{ct}_{8}^{-1} \cdot \mathrm{ct}_{8}^{\prime}=\mathbf{e}\left(K_{1}, K_{2}\right)^{s_{2}-s_{2}^{\prime}}=\mathbf{e}\left(K_{1}, K_{2}^{s_{2}}\right) \cdot \mathbf{e}\left(K_{1}^{-1}, K_{2}^{s_{2}^{\prime}}\right)= \\
& \mathbf{e}\left(K_{1}, \mathcal{K}_{2}\right) \cdot \mathbf{e}\left(K_{1}^{-1}, \mathcal{K}_{1}^{\prime}\right)
\end{aligned}
$$

And to prove that $\mathrm{ct}_{1}=g^{s_{2}}$ and $\mathrm{ct}_{8}=\lambda^{-s_{2}} \cdot m$, we add the following equation:

$$
\mathbf{e}\left(\mathrm{ct}_{1}, K_{1}\right)=\mathbf{e}\left(g, \mathcal{K}_{1}\right), \mathbf{e}\left(\mathrm{ct}_{1}^{\prime}, K_{1}\right)=\mathbf{e}\left(g, \mathcal{K}_{1}^{\prime}\right)
$$

So we have the following system of equations for one single ciphertext.

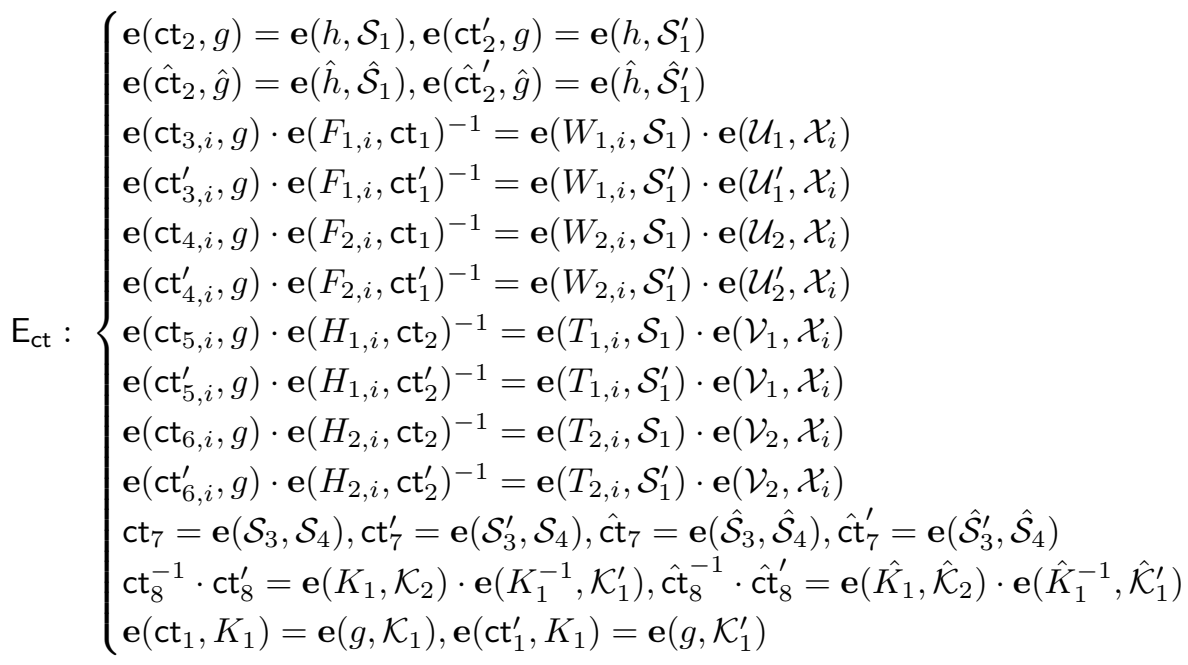


Now we need to provide a proof that two ciphertexts ct, $\hat{c t}$ are the encryption of a single message $m$ and a single attribute $\boldsymbol{x}$ :

$$
\mathcal{X}_{i}=g^{x_{i}}, \hat{\mathcal{X}}_{i}=\hat{g}^{x_{i}} \Rightarrow \mathbf{e}\left(\mathcal{X}_{i}, \hat{g}\right)=\mathbf{e}\left(g, \hat{\mathcal{X}}_{i}\right) \Rightarrow \mathbf{e}\left(\mathcal{X}_{i}, \hat{g}\right) \cdot \mathbf{e}\left(g, \hat{\mathcal{X}}_{i}\right)^{-1}=1_{\mathbb{G}_{T}}
$$

Notice that $\mathrm{ct}_{8}, \mathrm{ct}_{8}^{\prime}$ are the only components of the ciphertext which are related to the message, $m$, so we have:

$$
\begin{aligned}
& \left(\mathrm{ct}_{8}=\Lambda^{-s_{2}} \mathbf{m}, \hat{\mathrm{ct}}_{8}=\hat{\Lambda}^{-\hat{s}_{2}} \mathbf{m}\right) \Rightarrow \mathrm{ct}_{8} \hat{\mathrm{ct}}_{8}^{-1}=\Lambda^{-s_{2}} \cdot \hat{\Lambda}^{\hat{s}_{2}}= \\
& \mathbf{e}\left(K_{1}^{s_{2}}, K_{2}^{-1}\right) \cdot \mathbf{e}\left(\hat{K}_{1}^{\hat{s}_{2}}, \hat{K}_{2}\right)==\mathbf{e}\left(\mathcal{K}_{1}, K_{2}^{-1}\right) \cdot \mathbf{e}\left(\hat{\mathcal{K}}_{1}, \hat{K}_{2}\right)=\mathbf{e}\left(K_{1}^{-1}, \mathcal{K}_{2}\right) \cdot \mathbf{e}\left(\hat{K}_{1}, \hat{\mathcal{K}}_{2}\right)
\end{aligned}
$$

So the prover has to provide a proof for the following system of equations:

$$
\mathrm{E}_{\mathrm{ct}-\hat{\mathrm{ct}}}:\left\{\begin{array}{l}
\mathrm{ct}_{8} \hat{\mathrm{ct}}_{8}^{-1}=\mathbf{e}\left(\mathcal{K}_{1}, K_{2}^{-1},\right) \cdot \mathbf{e}\left(\hat{\mathcal{K}}_{1}, \hat{K}_{2}\right) \\
\mathrm{ct}_{8} \hat{\mathrm{ct}}_{8}^{-1}=\mathbf{e}\left(K_{1}^{-1}, \mathcal{K}_{2}\right) \cdot \mathbf{e}\left(\hat{K}_{1}, \hat{\mathcal{K}}_{2}\right) \\
\mathbf{e}\left(g, \mathcal{K}_{1}\right)=\mathbf{e}\left(\mathrm{ct}_{1}, K_{1}\right), \mathbf{e}\left(\hat{g}, \hat{\mathcal{K}}_{1}\right)=\mathbf{e}\left(\hat{\mathrm{ct}}_{1}, \hat{K}_{1}\right) \\
\mathbf{e}\left(\mathcal{X}_{i}, \hat{g}\right) \cdot \mathbf{e}\left(g, \hat{\mathcal{X}}_{i}\right)^{-1}=1_{\mathbb{G}_{T}}
\end{array}\right.
$$

Summing up, to provide the NIWI-proof system for encryption algorithm the prover uses Groth-Sahai proof-system for the system of equations, $E_{C T}=$ Ect $\wedge$ $\mathrm{E}_{\mathrm{ct}-\hat{\mathrm{ct}}}$.

\section{Conclusion}

Our main contribution is the first efficient verifiable (attribute-hiding) IPE scheme from bilinear groups. The privacy of our scheme is based on the standard DLIN assumption whereas its verifiability is unconditional. Towards this goal, we also constructed the first perfectly correct inner product encryption scheme for plaintexts of arbitrary length. Our VIPE scheme is selectively secure only; we leave as an interesting open problem the construction of a fully secure one.

Acknowledgments. We would like to thank the Luxembourg National Research Fund (FNR) for funding this reserach. In particular N. Soroush and V. Iovino were supported by the FNR CORE project FESS (no. C16/IS/11299247). A. Rial was supported by the FNR CORE project SZK (no. C17/11650748) and P. Roenne was supported by the INTER-SURCVS project.

\section{References}

1. Abdalla, M., et al.: Searchable encryption revisited: consistency properties, relation to anonymous IBE, and extensions. In: Shoup, V. (ed.) CRYPTO 2005. LNCS, vol. 3621, pp. 205-222. Springer, Heidelberg (2005). https://doi.org/10. 1007/11535218_13

2. Badrinarayanan, S., Goyal, V., Jain, A., Sahai, A.: Verifiable functional encryption. In: Cheon, J.H., Takagi, T. (eds.) ASIACRYPT 2016, Part II. LNCS, vol. 10032, pp. 557-587. Springer, Heidelberg (2016). https://doi.org/10.1007/978-3-662-53890$6 \_19$ 
3. Baltico, C.E.Z., Catalano, D., Fiore, D., Gay, R.: Practical functional encryption for quadratic functions with applications to predicate encryption. In: Katz, J., Shacham, H. (eds.) CRYPTO 2017, Part I. LNCS, vol. 10401, pp. 67-98. Springer, Cham (2017). https://doi.org/10.1007/978-3-319-63688-7_3

4. Boneh, D., Canetti, R., Halevi, S., Katz, J.: Chosen-ciphertext security from identity-based encryption. SIAM J. Comput. 36(5), 1301-1328 (2007)

5. Boneh, D., Di Crescenzo, G., Ostrovsky, R., Persiano, G.: Public key encryption with keyword search. In: Cachin, C., Camenisch, J.L. (eds.) EUROCRYPT 2004. LNCS, vol. 3027, pp. 506-522. Springer, Heidelberg (2004). https://doi.org/10. 1007/978-3-540-24676-3_30

6. Boneh, D., Franklin, M.K.: Identity-based encryption from the Weil pairing. In: Kilian, J. (ed.) CRYPTO 2001. LNCS, vol. 2139, pp. 213-229. Springer, Heidelberg (2001). https://doi.org/10.1007/3-540-44647-8_13

7. Boneh, D., Sahai, A., Waters, B.: Functional encryption: definitions and challenges. In: Ishai, Y. (ed.) TCC 2011. LNCS, vol. 6597, pp. 253-273. Springer, Heidelberg (2011). https://doi.org/10.1007/978-3-642-19571-6_16

8. Boneh, D., Waters, B.: Conjunctive, subset, and range queries on encrypted data. In: Vadhan, S.P. (ed.) TCC 2007. LNCS, vol. 4392, pp. 535-554. Springer, Heidelberg (2007). https://doi.org/10.1007/978-3-540-70936-7_29

9. Cocks, C.: An identity based encryption scheme based on quadratic residues. In: Honary, B. (ed.) Cryptography and Coding 2001. LNCS, vol. 2260, pp. 360-363. Springer, Heidelberg (2001). https://doi.org/10.1007/3-540-45325-3_32

10. De Caro, A., Iovino, V.: On the power of rewinding simulators in functional encryption. Des. Codes Cryptogr. 84(3), 373-399 (2016). https://doi.org/10.1007/s10623016-0272-x

11. De Caro, A., Iovino, V., Jain, A., O’Neill, A., Paneth, O., Persiano, G.: On the achievability of simulation-based security for functional encryption. In: Canetti, R., Garay, J.A. (eds.) CRYPTO 2013, Part II. LNCS, vol. 8043, pp. 519-535. Springer, Heidelberg (2013). https://doi.org/10.1007/978-3-642-40084-1_29

12. Groth, J.: Simulation-sound NIZK proofs for a practical language and constant size group signatures. In: Lai, X., Chen, K. (eds.) ASIACRYPT 2006. LNCS, vol. 4284, pp. 444-459. Springer, Heidelberg (2006). https://doi.org/10.1007/11935230_29

13. Groth, J., Ostrovsky, R., Sahai, A.: Non-interactive zaps and new techniques for NIZK. In: Dwork, C. (ed.) CRYPTO 2006. LNCS, vol. 4117, pp. 97-111. Springer, Heidelberg (2006). https://doi.org/10.1007/11818175_6

14. Groth, J., Sahai, A.: Efficient non-interactive proof systems for bilinear groups. In: Smart, N. (ed.) EUROCRYPT 2008. LNCS, vol. 4965, pp. 415-432. Springer, Heidelberg (2008). https://doi.org/10.1007/978-3-540-78967-3_24

15. Iovino, V., Żebroski, K.: Simulation-based secure functional encryption in the random oracle model. In: Lauter, K., Rodríguez-Henríquez, F. (eds.) LATINCRYPT 2015. LNCS, vol. 9230, pp. 21-39. Springer, Cham (2015). https://doi.org/10.1007/ 978-3-319-22174-8_2

16. Ishai, Y., Kushilevitz, E., Ostrovsky, R., Sahai, A.: Zero-knowledge from secure multiparty computation. In: Proceedings of the 39th Annual ACM Symposium on Theory of Computing, San Diego, California, USA, 11-13 June 2007, pp. 21-30 (2007)

17. Kate, A., Zaverucha, G.M., Goldberg, I.: Constant-size commitments to polynomials and their applications. In: Abe, M. (ed.) ASIACRYPT 2010. LNCS, vol. 6477, pp. 177-194. Springer, Heidelberg (2010). https://doi.org/10.1007/978-3642-17373-8_11 
18. Katz, J., Sahai, A., Waters, B.: Predicate encryption supporting disjunctions, polynomial equations, and inner products. In: Smart, N. (ed.) EUROCRYPT 2008. LNCS, vol. 4965, pp. 146-162. Springer, Heidelberg (2008). https://doi.org/10. 1007/978-3-540-78967-3_9

19. Lewko, A.B., Okamoto, T., Sahai, A., Takashima, K., Waters, B.: Fully secure functional encryption: attribute-based encryption and (hierarchical) inner product encryption. In: Gilbert, H. (ed.) EUROCRYPT 2010. LNCS, vol. 6110, pp. 62-91. Springer, Heidelberg (2010). https://doi.org/10.1007/978-3-642-13190-5_4

20. Libert, B., Ramanna, S.C., Yung, M.: Functional commitment schemes: from polynomial commitments to pairing-based accumulators from simple assumptions. In: 43rd International Colloquium on Automata, Languages, and Programming, ICALP 2016, 11-15 July 2016, Rome, Italy, pp. 30:1-30:14 (2016)

21. Okamoto, T., Takashima, K.: Adaptively attribute-hiding (hierarchical) inner product encryption. In: Pointcheval, D., Johansson, T. (eds.) EUROCRYPT 2012. LNCS, vol. 7237, pp. 591-608. Springer, Heidelberg (2012). https://doi.org/10. 1007/978-3-642-29011-4_35

22. Park, J.H.: Inner-product encryption under standard assumptions. Des. Codes Cryptogr. 58(3), 235-257 (2011). https://doi.org/10.1007/s10623-010-9405-9

23. Sahai, A., Waters, B.: Fuzzy identity-based encryption. In: Cramer, R. (ed.) EUROCRYPT 2005. LNCS, vol. 3494, pp. 457-473. Springer, Heidelberg (2005). https:// doi.org/10.1007/11426639_27

24. Shamir, A.: Identity-based cryptosystems and signature schemes. In: Blakley, G.R., Chaum, D. (eds.) CRYPTO 1984. LNCS, vol. 196, pp. 47-53. Springer, Heidelberg (1985). https://doi.org/10.1007/3-540-39568-7_5

25. Soroush, N., Iovino, V., Rial, A., Roenne, P.B., Ryan, P.Y.A.: Verifiable inner product encryption scheme. Cryptology ePrint Archive, Report 2020/122 (2020). https://eprint.iacr.org/2020/122

26. Tang, Q., Ji, D.: Verifiable attribute-based encryption. IJ Netw. Secur. 10(2), 114$120(2010)$ 